\title{
On rough maximal operators and Marcinkiewicz integrals along submanifolds
}

by

H. M. Al-QAssem (Doha) and Y. PAN (Pittsburgh, PA)

\begin{abstract}
We investigate the $L^{p}$ boundedness for a class of parametric Marcinkiewicz integral operators associated to submanifolds and a class of related maximal operators under the $L(\log L)^{\alpha}\left(\mathbb{S}^{n-1}\right)$ condition on the kernel functions. Our results improve and extend some known results.
\end{abstract}

1. Introduction and statement of results. Let $\mathbb{R}^{n}(n \geq 2)$ be the $n$-dimensional Euclidean space and $\mathbb{S}^{n-1}$ be the unit sphere in $\mathbb{R}^{n}$ equipped with the induced Lebesgue measure $d \sigma=d \sigma(\cdot)$. For $x \in \mathbb{R}^{n} \backslash\{0\}$, let $x^{\prime}=x /|x|$. Let $\Omega$ be a function in $L^{1}\left(\mathbb{S}^{n-1}\right)$ satisfying

$$
\int_{\mathbb{S}^{n-1}} \Omega(x) d \sigma(x)=0 .
$$

For $1 \leq \gamma \leq \infty$, let $\Delta_{\gamma}\left(\mathbb{R}_{+}\right)$denote the collection of all measurable functions $h:[0, \infty) \rightarrow \mathbb{C}$ satisfying $\sup _{R>0}\left(R^{-1} \int_{0}^{R}|h(t)|^{\gamma} d t\right)^{1 / \gamma}<\infty$. We note that

$$
\begin{gathered}
L^{\infty}\left(\mathbb{R}_{+}\right) \subset \Delta_{\beta}\left(\mathbb{R}_{+}\right) \subset \Delta_{\alpha}\left(\mathbb{R}_{+}\right) \quad \text { for } \alpha<\beta, \\
L^{\gamma}\left(\mathbb{R}_{+}, d t / t\right) \subset \Delta_{\gamma}\left(\mathbb{R}_{+}\right) \quad \text { for } 1 \leq \gamma<\infty,
\end{gathered}
$$

and all these inclusions are proper. Let $L(\log L)^{\alpha}\left(\mathbb{S}^{n-1}\right)$ (for $\left.\alpha>0\right)$ denote the class of all measurable functions $\Omega$ on $\mathbb{S}^{n-1}$ which satisfy

$$
\|\Omega\|_{L(\log L)^{\alpha}\left(\mathbb{S}^{n-1}\right)}=\int_{\mathbb{S}^{n-1}}|\Omega(y)| \log ^{\alpha}(2+|\Omega(y)|) d \sigma(y)<\infty .
$$

In this paper, we are interested in parametric Marcinkiewicz integral operators of the form

2000 Mathematics Subject Classification: Primary 42B20; Secondary 42B15, 42B25.

Key words and phrases: Marcinkiewicz integrals, maximal operators, $L^{p}$ boundedness, rough kernel.

The work on this paper was done while the first author was on sabbatical leave from Yarmouk University. 


$$
\begin{aligned}
& \mathcal{M}_{\Omega, \phi, \psi, h}^{\varrho} f\left(x, x_{n+1}\right) \\
& \quad=\left(\int_{0}^{\infty}\left|t^{-\varrho} \int_{|u| \leq t} f\left(x-\phi(|u|) u^{\prime}, x_{n+1}-\psi(|u|)\right) \frac{\Omega\left(u^{\prime}\right)}{|u|^{n-\varrho}} h(|u|) d u\right|^{2} \frac{d t}{t}\right)^{1 / 2},
\end{aligned}
$$

where $\left(x, x_{n+1}\right) \in \mathbb{R}^{n} \times \mathbb{R}=\mathbb{R}^{n+1}, \phi$ and $\psi$ are suitable functions defined on $\mathbb{R}_{+}, \varrho=\sigma+i \tau(\sigma, \tau \in \mathbb{R}$ with $\sigma>0)$ and $f \in \mathcal{S}\left(\mathbb{R}^{n+1}\right)$, the space of Schwartz functions.

When $\phi(t) \equiv t$ and $\psi \equiv 0$, we denote $\mathcal{M}_{\Omega, \phi, \psi, h}^{\varrho}$ by $\mathcal{M}_{\Omega, h}^{\varrho}$. In the special case of $\varrho=1$ and $h=1, \mathcal{M}_{\Omega, h}^{\varrho}$ is essentially the classical Marcinkiewicz integral operator

$$
\mathcal{M}_{\Omega} f(x)=\left(\int_{0}^{\infty}\left|\int_{|u| \leq t} f(x-u) \frac{\Omega\left(u^{\prime}\right)}{|u|^{n-1}} d u\right|^{2} \frac{d t}{t^{3}}\right)^{1 / 2},
$$

which was introduced by E. M. Stein as a higher dimensional analogue of

$$
f \mapsto\left(\int_{0}^{2 \pi}|F(\theta+t)+F(\theta-t)-2 F(\theta)|^{2} \frac{d t}{t^{3}}\right)^{1 / 2},
$$

where $F(\theta)=\int_{0}^{\theta} f(s) d s+C$. Such operators belong to the broad class of Littlewood-Paley $g$-functions, and $L^{p}$ bounds for them are useful in the study of smoothness properties of functions and behavior of integral transformations, such as Poisson integrals, singular integrals and, more generally, singular Radon transforms. The readers are referred to [Di], [St1], [Sa], [TW], $[\mathrm{DFP}],[\mathrm{SY}],[\mathrm{AA}]$, and $[\mathrm{AACP}]$ for a survey of past studies as well as some of the more recent advances on this topic. Below we shall recall a few known results which are directly relevant to our current study.

We start with the following result obtained in $[\mathrm{AACP}]$ :

TheOREM A. If $\Omega \in L(\log L)^{1 / 2}\left(\mathbb{S}^{n-1}\right)$, then $\mathcal{M}_{\Omega}$ is bounded on $L^{p}\left(\mathbb{R}^{n}\right)$ for $1<p<\infty$. Moreover, the exponent $1 / 2$ is the best possible.

We point out that $\mathrm{T}$. Walsh [Wa] proved the result in Theorem $\mathrm{A}$ for $p=2$ and also that the exponent $1 / 2$ in $L(\log L)^{1 / 2}\left(\mathbb{S}^{n-1}\right)$ cannot be replaced by any smaller number. As for the parametric Marcinkiewicz operator $\mathcal{M}_{\Omega, h}^{\varrho}$, Hörmander [Ho] proved that if $h(r) \equiv 1, \varrho>0$, and $\Omega \in \operatorname{Lip}_{\alpha}\left(\mathbb{S}^{n-1}\right)$ with $\alpha>0$, then $\mathcal{M}_{\Omega, h}^{\varrho}$ is bounded on $L^{p}\left(\mathbb{R}^{n}\right)$ for $p \in(1, \infty)$. Sakamoto and Yabuta $[\mathrm{SY}]$ proved that $\mathcal{M}_{\Omega, h}^{\varrho}$ is bounded on $L^{p}$ for $p \in(1, \infty)$ if $h(r) \equiv 1$, $\Omega \in \operatorname{Lip}_{\alpha}\left(\mathbb{S}^{n-1}\right)$ with $\alpha>0, h(r) \equiv 1$ and $\varrho$ is complex with $\operatorname{Re}(\varrho)>0$. In [DLY] the authors improved the result in [SY] as described in the following theorem:

THEOREM B. If $h \in \Delta_{\gamma}\left(\mathbb{R}_{+}\right)$for some $\gamma>1$ and $\Omega \in L(\log L)\left(\mathbb{S}^{n-1}\right)$, then $\mathcal{M}_{\Omega, h}^{\varrho}$ is bounded on $L^{2}\left(\mathbb{R}^{n}\right)$. 
If $\phi(t) \equiv t, \varrho=1$ and $\psi$ is a suitable function, then $\mathcal{M}_{\Omega, \phi, \psi, h}^{\varrho}$ (denoted by $\mathcal{M}_{\Omega, \mathbf{1}, \psi, h}^{1}$ ) is the Marcinkiewicz integral operator along the surface of revolution $S_{\psi}=\left\{(y, \psi(|y|)): y \in \mathbb{R}^{n}\right\}$ studied by Ding-Fan-Pan in [DFP]. We point out that the study of the related singular integrals along surfaces of revolution was initiated by Kim, Wainger, Wright and Ziesler [KWWZ] and continued by many authors (see, for example, [AP2], $[\mathrm{AsP}]$ and the references therein). In $[\mathrm{AA}]$, the authors proved the following result:

Theorem C. Suppose that $\psi: \mathbb{R}_{+} \rightarrow \mathbb{R}_{+}$is a $C^{2}$, convex, increasing function with $\psi(0)=0$, and $h \in \Delta_{\gamma}\left(\mathbb{R}_{+}\right)$for some $\gamma>1$. If $\varrho=1$ and $\Omega \in L(\log L)\left(\mathbb{S}^{n-1}\right)$ satisfies $(1.1)$, then $\mathcal{M}_{\Omega, \mathbf{1}, \psi, h}^{1}$ is bounded on $L^{p}\left(\mathbb{R}^{n+1}\right)$ for $|1 / p-1 / 2|<\min \left\{1 / 2,1 / \gamma^{\prime}\right\}$

If $\psi(t) \equiv 0, \varrho=1$ and $\phi$ is a suitable function, then $\mathcal{M}_{\Omega, \phi, \psi, h}^{\varrho}$ (denote it by $\left.\mathcal{M}_{\Omega, \phi, \mathbf{0}, h}^{1}\right)$ is the Marcinkiewicz integral operator studied by Al-Qassem [A1] and described in the following:

Theorem D. Let $h \in \Delta_{\gamma}\left(\mathbb{R}_{+}\right)$for some $\gamma>1$. Let $\phi$ be a $C^{2}([0, \infty))$, convex, increasing function with $\phi(0)=0$. If $\Omega \in L(\log L)^{1 / 2}\left(\mathbb{S}^{n-1}\right)$ satisfies (1.1), then $\mathcal{M}_{\Omega, \phi, \mathbf{0}, h}^{1}$ is bounded on $L^{p}\left(\mathbb{R}^{n}\right)$ for $|1 / p-1 / 2|<\min \left\{1 / 2,1 / \gamma^{\prime}\right\}$.

We remark that the study of the $L^{p}$ boundedness of the corresponding singular integral operator (when $\psi \equiv 0$ and $\phi$ is a suitable function) was initiated by Fan-Pan in [FP2] and continued by many authors (see, for example, [AP2]).

We notice that the results in Theorems $\mathrm{B}$ and $\mathrm{C}$ fall short of what is known regarding the classical operator $\mathcal{M}_{\Omega}$ because $L(\log L)^{1 / 2}\left(\mathbb{S}^{n-1}\right) \subseteq$ $L(\log L)\left(\mathbb{S}^{n-1}\right)$, and that the range of $p$ given in both Theorems $\mathrm{C}$ and $\mathrm{D}$ is the entire range $(1, \infty)$ whenever $\gamma \geq 2$, whereas this range becomes progressively smaller as $\gamma \rightarrow 1^{+}$. For relevant results on Marcinkiewicz integrals and singular integrals having such limited range of $p$, we refer the readers to [FP1], [FP2], [AsP], [AP1], and [AP2]. In light of the results cited above, the following problems arise naturally:

Problem.

(1) Determine whether the operators $\mathcal{M}_{\Omega, \mathbf{1}, \psi, h}^{1}$ and $\mathcal{M}_{\Omega, \phi, \mathbf{0}, h}^{1}$ can be bounded on $L^{p}$ for $p$ outside the range $|1 / p-1 / 2|<\min \left\{1 / 2,1 / \gamma^{\prime}\right\}$.

(2) Determine whether the operator $\mathcal{M}_{\Omega, \mathbf{1}, \psi, h}^{1}$ is bounded on $L^{p}$ under the natural condition $\Omega \in L(\log L)^{1 / 2}\left(\mathbb{S}^{n-1}\right)$.

In the current paper we are primarily concerned with a solution to the above problem. The main results of this paper are stated in the following theorems: 
Theorem 1.1. Let $h \in \Delta_{\gamma}\left(\mathbb{R}_{+}\right)$for $1<\gamma \leq \infty$. Assume that $\phi$ and $\psi$ are $C^{2}([0, \infty))$, convex, increasing functions with $\phi(0)=\psi(0)=0$.

(a) If $\Omega \in L(\log L)^{1 / 2}\left(\mathbb{S}^{n-1}\right)$, then the operator $\mathcal{M}_{\Omega, \phi, \psi, h}^{\varrho}$ is bounded on $L^{p}\left(\mathbb{R}^{n+1}\right)$ for $2 \leq p<1 /(1 / 2-\alpha(\gamma))$, where $\alpha(\gamma)=\min \left\{1 / 2,1 / \gamma^{\prime}\right\}$.

(b) If $\Omega \in L(\log L)^{1 / \gamma^{\prime}}\left(\mathbb{S}^{n-1}\right)$ with $2<\gamma \leq \infty$, then $\mathcal{M}_{\Omega, \phi, \psi, h}^{\varrho}$ is bounded on $L^{p}\left(\mathbb{R}^{n+1}\right)$ for $\gamma^{\prime}<p<\infty$.

(c) If $\Omega \in L(\log L)^{(2 \gamma-1) /(2 \gamma)}\left(\mathbb{S}^{n-1}\right)$ with $1<\gamma \leq 2$, then $\mathcal{M}_{\Omega, \phi, \psi, h}^{\varrho}$ is bounded on $L^{p}\left(\mathbb{R}^{n+1}\right)$ for $2 \gamma /(2 \gamma-1)<p<2$.

(d) If $\Omega \in L(\log L)^{(3 \gamma-2) /(2 \gamma)}\left(\mathbb{S}^{n-1}\right)$ with $1<\gamma \leq 2$, then $\mathcal{M}_{\Omega, \phi, \psi, h}^{\varrho}$ is bounded on $L^{p}\left(\mathbb{R}^{n+1}\right)$ for $2 \gamma /(3 \gamma-2)<p<2$.

Notice that in (a)-(b), $L(\log L)^{1 / \gamma^{\prime}}\left(\mathbb{S}^{n-1}\right) \subseteq L(\log L)^{1 / 2}\left(\mathbb{S}^{n-1}\right)$ if $2<$ $\gamma \leq \infty$, but the range of $p$ in (b) is better than in (a). Also, in (c)-(d), $L(\log L)^{(2 \gamma-1) /(2 \gamma)}\left(\mathbb{S}^{n-1}\right) \subseteq L(\log L)^{(3 \gamma-2) /(2 \gamma)}\left(\mathbb{S}^{n-1}\right)$, but the range of $p$ in (d) is better than in (c). If we impose a more restrictive condition on $h$ we have the following sharper result with respect to the condition on $\Omega$ and the range of $p$ :

Theorem 1.2. Suppose that $h \in L^{\gamma}\left(\mathbb{R}_{+}, d t / t\right)$ with $1 \leq \gamma \leq \infty$. Assume that $\phi$ and $\psi$ are $C^{2}([0, \infty))$, convex, increasing functions with $\phi(0)=$ $\psi(0)=0$.

(a) If $\Omega \in L(\log L)^{1 / \gamma^{\prime}}\left(\mathbb{S}^{n-1}\right)$ with $1<\gamma \leq 2$, then $\mathcal{M}_{\Omega, \phi, \psi, h}^{\varrho}$ is bounded on $L^{p}\left(\mathbb{R}^{n+1}\right)$ for $\gamma^{\prime} \leq p<\infty$.

(b) If $\Omega \in L(\log L)^{1 / 2}\left(\mathbb{S}^{n-1}\right)$ and $2<\gamma \leq \infty$, then $\mathcal{M}_{\Omega, \phi, \psi, h}^{\varrho}$ is bounded on $L^{p}\left(\mathbb{R}^{n+1}\right)$ for $2 \leq p<\infty$.

(c) If $\gamma=1$ and $\Omega \in L^{1}\left(\mathbb{S}^{n-1}\right)$, then $\mathcal{M}_{\Omega, \phi, \psi, h}^{\varrho}$ is bounded on $L^{\infty}\left(\mathbb{R}^{n+1}\right)$.

At this point, we remark that our results cannot be proved by application of existing arguments on Marcinkiewicz integrals and some new maximal functions must be introduced. One of these maximal functions, which is related to Marcinkiewicz integrals, is

$$
M_{\phi, \psi, \Omega}^{(\gamma)} f\left(x, x_{n+1}\right)=\sup _{h}\left|\mathcal{M}_{\Omega, \phi, \psi, h}^{\varrho} f\left(x, x_{n+1}\right)\right|,
$$

where the supremum is taken over all measurable radial functions $h$ with $\|h\|_{L^{\gamma}\left(\mathbb{R}_{+}, d t / t\right)} \leq 1$. If $\phi(t) \equiv t, \psi \equiv 0$ and $\varrho=1$, we denote $M_{\phi, \psi, \Omega}^{(\gamma)}$ by $M_{\Omega}^{(\gamma)}$. We remark that the definition of $M_{\Omega}^{(\gamma)}$ is motivated by the definition of the maximal operator $\Im_{\Omega}^{(\gamma)}$ related to homogeneous singular integrals and defined by

$$
\Im_{\Omega}^{(\gamma)} f(x)=\left.\sup _{\|h\|_{L^{\gamma}\left(\mathbb{R}_{+}, d t / t\right)} \leq 1}\left|\int_{\mathbb{R}^{n}} f(x-y) h(|y|) \Omega\left(y^{\prime}\right)\right| y\right|^{-n} d y \mid .
$$


The operator $\Im_{\Omega}^{(\gamma)}$ was formally introduced by L. K. Chen and H. Lin in their work on singular integrals [CL]. We refer the readers to [Le], [A2], [As] for the importance and applications of this maximal operator. Our result regarding the maximal operator $M_{\phi, \psi, \Omega}^{(\gamma)}$ is the following:

TheOREM 1.3. Assume that $\phi$ and $\psi$ are $C^{2}([0, \infty))$, convex, increasing functions with $\phi(0)=\psi(0)=0$.

(a) If $\Omega \in L(\log L)^{1 / \gamma^{\prime}}\left(\mathbb{S}^{n-1}\right)$ satisfies (1.1), then $M_{\phi, \psi, \Omega}^{(\gamma)}$ is bounded on $L^{p}\left(\mathbb{R}^{n+1}\right)$ for $\gamma^{\prime} \leq p<\infty$ and $1<\gamma \leq 2$.

(b) If $\Omega \in L(\log L)^{1 / 2}\left(\mathbb{S}^{n-1}\right)$ satisfies (1.1), then $M_{\phi, \psi, \Omega}^{(\gamma)}$ is bounded on $L^{p}\left(\mathbb{R}^{n+1}\right)$ for $2 \leq p<\infty$ and $2<\gamma \leq \infty$.

(c) $M_{\phi, \psi, \Omega}^{(\gamma)}$ is bounded on $L^{\infty}\left(\mathbb{R}^{n+1}\right)$ if $\gamma=1$ and $\Omega \in L^{1}\left(\mathbb{S}^{n-1}\right)$.

(d) There exists an $\Omega$ which lies in $L(\log L)^{1 / 2-\varepsilon}\left(\mathbb{S}^{n-1}\right)$ for all $\varepsilon>0$ and satisfies (1.1) such that $M_{\Omega}^{(2)}$ is not bounded on $L^{2}\left(\mathbb{R}^{n}\right)$.

Remarks. (1) To clarify the above results, we remark that, for any $q>1$, the following proper inclusions hold:

$$
\begin{aligned}
L^{q}\left(\mathbb{S}^{n-1}\right) & \subset L(\log L)\left(\mathbb{S}^{n-1}\right) \subset H^{1}\left(\mathbb{S}^{n-1}\right) \subset L^{1}\left(\mathbb{S}^{n-1}\right), \\
L(\log L)^{\beta}\left(\mathbb{S}^{n-1}\right) & \subset L(\log L)^{\alpha}\left(\mathbb{S}^{n-1}\right) \quad \text { if } 0<\alpha<\beta \\
L(\log L)^{\alpha}\left(\mathbb{S}^{n-1}\right) & \subset H^{1}\left(\mathbb{S}^{n-1}\right) \quad \text { for all } \alpha \geq 1
\end{aligned}
$$

while

$$
L(\log L)^{\alpha}\left(\mathbb{S}^{n-1}\right) \nsubseteq H^{1}\left(\mathbb{S}^{n-1}\right) \nsubseteq L L(\log L)^{\alpha}\left(\mathbb{S}^{n-1}\right) \quad \text { for all } 0<\alpha<1 .
$$

Here $H^{1}\left(\mathbb{S}^{n-1}\right)$ is the Hardy space on the unit sphere.

(2) For the case $h \in L^{\infty}\left(\mathbb{R}_{+}\right)$, the authors of [AAFJ] showed that there is a function $f \in L^{p}$ such that the maximal operator related to singular integrals acting on $f$ (i.e. $\Im_{\Omega}^{(\infty)}(f)$ ) yields an identically infinite function. It is still unknown whether $\Im_{\Omega}^{(\gamma)}$ is bounded on $2<\gamma<\infty$. It is worth noting that the maximal operator related to the Marcinkiewicz integrals $\mathcal{M}_{\Omega, \phi, \psi, h}^{\varrho}$ is bounded on $L^{p}$ even if $2<\gamma \leq \infty$.

(3) We notice that the Marcinkiewicz operator $\mathcal{M}_{\Omega, h}^{1}$ is bounded on $L^{p}$ if $\Omega \in L(\log L)^{1 / \gamma^{\prime}}\left(\mathbb{S}^{n-1}\right) \subset L(\log L)^{1 / 2}\left(\mathbb{S}^{n-1}\right)$ and $h \in L^{\gamma}\left(\mathbb{R}_{+}, d t / t\right)(1<$ $\gamma \leq 2$ ), while the classical Marcinkiewicz operator $\mathcal{M}_{\Omega}$ is bounded on $L^{p}$ if $\Omega \in L(\log L)^{1 / 2}\left(\mathbb{S}^{n-1}\right)$. Since the condition $\Omega \in L(\log L)^{1 / 2}\left(\mathbb{S}^{n-1}\right)$ is known to be the best possible for the $L^{2}$ boundedness of $\mathcal{M}_{\Omega}$ to hold, this reveals that the Marcinkiewicz integral operators $\mathcal{M}_{\Omega, h}^{1}$ (with $h \in L^{\gamma}\left(\mathbb{R}_{+}, d t / t\right)$ and $1<\gamma<\infty$ ) have weaker singularities than the classical Marcinkiewicz integral operator $\mathcal{M}_{\Omega}$ due to the presence of the strong condition on $h$. Also, this is evidenced by the fact $\mathcal{M}_{\Omega}$ is not bounded on $L^{\infty}\left(\mathbb{R}^{n}\right)$, while $\mathcal{M}_{\Omega, h}^{1}$ is bounded on $L^{\infty}\left(\mathbb{R}^{n}\right)$ if $h \in L^{1}\left(\mathbb{R}_{+}, d t / t\right)$. 
(4) Theorems 1.1 and 1.2 improve and extend Theorems A-D.

(5) If $h \in \Delta_{\gamma}\left(\mathbb{R}_{+}\right)(\gamma \geq 2)$, then Theorem 1.1(a) implies that $\mathcal{M}_{\Omega, \phi, \psi, h}^{\varrho}$ is bounded on $L^{p}$ for $2 \leq p<\infty$ under the condition $\Omega \in L(\log L)^{1 / 2}\left(\mathbb{S}^{n-1}\right)$. Also, if $\gamma=2$, then Theorem 1.1(c) and (d) respectively imply that $\mathcal{M}_{\Omega, \phi, \psi, h}^{\varrho}$ is bounded on $L^{p}$ for $4 / 3<p<2$ and $\Omega \in L(\log L)^{3 / 4}\left(\mathbb{S}^{n-1}\right)$, and for $1<p<2$ and $\Omega \in L(\log L)\left(\mathbb{S}^{n-1}\right)$. These results improve and extend Theorem $\mathrm{C}$ and a result in $[\mathrm{As}]$.

(6) The main tools used in this paper come from [A1], [A2], [Le], [DR] and [FP1].

Throughout the paper the letter $C$ will stand for a positive constant which is independent of the main parameters and not necessarily the same at each occurrence.

The authors wish to thank the referee for his helpful comments.

2. Proof of Theorem 1.3(d). Let $\chi_{A}$ denote the characteristic function of a set $A$. By definition of $M_{\Omega}^{(2)} f(x)$ and switching to polar coordinates we have

$$
M_{\Omega}^{(2)} f(x)=\sup _{\|h\|_{L^{2}\left(\mathbb{R}_{+}, d t / t\right)} \leq 1}\left(\int_{0}^{\infty}\left|\int_{0}^{\infty}\left(h(s) \chi_{[0, t]}(s)\right) A_{s, \Omega} f(x) d s\right|^{2} \frac{d t}{t^{3}}\right)^{1 / 2},
$$

where

$$
A_{s, \Omega} f(x)=\int_{\mathbb{S}^{n-1}} f(x-s y) \Omega(y) d \sigma(y) .
$$

By duality and a change of variable we have

$$
M_{\Omega}^{(2)} f(x)=\left(\int_{0}^{\infty}\left(\int_{0}^{1}\left|\int_{\mathbb{S}^{n-1}} f(x-s t y) \Omega(y) d \sigma(y)\right|^{2} s d s\right) \frac{d t}{t}\right)^{1 / 2} .
$$

It is clear that $M_{\Omega}^{(2)}$ is bounded on $L^{2}\left(\mathbb{R}^{n}\right)$ if and only if the function

$$
m(\xi)=\left(\int_{0}^{\infty} \int_{0}^{1}\left|\int_{\mathbb{S}^{n-1}} e^{-2 \pi i t s \xi \cdot u} \Omega(u) d \sigma(u)\right|^{2} \frac{s d s d t}{t}\right)^{1 / 2}
$$

is an $L^{\infty}$ function. It is easy to see that

$$
(m(\xi))^{2}=\lim _{\substack{N \rightarrow \infty \\ \varepsilon \rightarrow 0}} \int_{\mathbb{S}^{n-1} \times \mathbb{S}^{n-1}} \Omega(x) \overline{\Omega(y)} \int_{0}^{1} \int_{\varepsilon|\xi|}^{N|\xi|} e^{-2 \pi i t \xi^{\prime} \cdot(x-y)} \frac{d t}{t} s d s d \sigma(x) d \sigma(y) .
$$


Notice that

$$
\begin{aligned}
\int_{\varepsilon|\xi|}^{N|\xi|}\left(e^{-2 \pi i t s \xi^{\prime} \cdot(x-y)}-\right. & \cos (2 \pi t)) \frac{d t}{t} \\
& \rightarrow \log \left|\xi^{\prime} \cdot(s x-s y)\right|^{-1}-i \frac{\pi}{2} \operatorname{sgn}\left(\xi^{\prime} \cdot(s x-s y)\right)
\end{aligned}
$$

as $N \rightarrow \infty$ and $\varepsilon \rightarrow 0$, and the integral is bounded, uniformly in $\varepsilon$ and $N$, by $C\left(1+|\log | \xi^{\prime} \cdot(s x-s y)||\right)$. Therefore, using (1.1) and the Lebesgue dominated convergence theorem we obtain

$$
\begin{aligned}
(m(\xi))^{2} & =\int_{\mathbb{S}^{n-1} \times \mathbb{S}^{n-1}} \Omega(x) \overline{\Omega(y)} \\
& \times\left(\int_{0}^{1}\left(\log \left|\xi^{\prime} \cdot(s y-s x)\right|^{-1}-i \frac{\pi}{2} \operatorname{sgn}\left(\xi^{\prime} \cdot(s y-s x)\right)\right) s d s\right) d \sigma(x) d \sigma(y) .
\end{aligned}
$$

If $\Omega$ is a real-valued function, we have

$$
(m(\xi))^{2}=\int_{\mathbb{S}^{n-1} \times \mathbb{S}^{n-1}} \Omega(x) \Omega(y)\left(\int_{0}^{1}\left(\log \left|\xi^{\prime} \cdot(s y-s x)\right|^{-1}\right) s d s\right) d \sigma(x) d \sigma(y) .
$$

Now, for a nonzero real number $a$, it is easy to see that

$$
\int_{[0,1]} \log |s a|^{-1} s d s=\frac{1}{2} \log |a|^{-1}+1 / 4 \text {. }
$$

Therefore, by the cancellation condition on $\Omega$, we immediately get

$$
(m(\xi))^{2}=\frac{1}{4} \int_{\mathbb{S}^{n-1} \times \mathbb{S}^{n-1}} \Omega(x) \Omega(y)\left(\log \left|\xi^{\prime} \cdot(y-x)\right|^{-1}\right) d \sigma(x) d \sigma(y) .
$$

Now the rest of the proof follows by the same argument as in [AA2] and [As]. We omit the details.

\section{Some definitions and lemmas}

Definition 3.1. For arbitrary functions $\phi(\cdot)$ and $\psi(\cdot)$ on $\mathbb{R}_{+}$, a measurable function $h: \mathbb{R}_{+} \rightarrow \mathbb{C}$ and $\Omega_{m}: \mathbb{S}^{n-1} \rightarrow \mathbb{R}$ with $m \in \mathbb{N} \cup\{0\}$, we define the family $\left\{\sigma_{t, m, h}: t \in \mathbb{R}_{+}\right\}$of measures and the maximal operator $\sigma_{m, h}^{*}$ on $\mathbb{R}^{n+1}$ by

$$
\begin{aligned}
\int_{\mathbb{R}^{n+1}} f d \sigma_{t, m, h} & =\frac{1}{t^{\varrho}} \int_{t / 2<|u| \leq t} f\left(\phi(|u|) u^{\prime}, \psi(|u|)\right) h(|u|) \frac{\Omega_{m}\left(u^{\prime}\right)}{|u|^{n-\varrho}} d u, \\
\sigma_{m, h}^{*} f\left(x, x_{n+1}\right) & =\sup _{t \in \mathbb{R}_{+}}|| \sigma_{t, m, h}\left|* f\left(x, x_{n+1}\right)\right|,
\end{aligned}
$$

where $\left|\sigma_{t, m, h}\right|$ is defined in the same way as $\sigma_{t, m, h}$, but with $\Omega_{m}$ replaced by $\left|\Omega_{m}\right|$ and $h$ replaced by $|h|$. 
LEMma 3.2. Let $m \in \mathbb{N} \cup\{0\}, a_{m}=2^{m+1}, \psi(\cdot)$ be an arbitrary function on $\mathbb{R}_{+}$, and $h \in \Delta_{\gamma}\left(\mathbb{R}_{+}\right)$for some $1<\gamma \leq \infty$. Let $\Omega_{m}$ be a function on $\mathbb{S}^{n-1}$ such that:

(i) $\left\|\Omega_{m}\right\|_{L^{1}\left(\mathbb{S}^{n-1}\right)} \leq 1$,

(ii) $\left\|\Omega_{m}\right\|_{L^{2}\left(\mathbb{S}^{n-1}\right)} \leq a_{m}^{2}$,

(iii) $\Omega_{m}$ satisfies (1.1) with $\Omega$ replaced by $\Omega_{m}$.

Assume that $\phi$ is a $C^{2}([0, \infty))$, convex, increasing function with $\phi(0)=0$. Then there exist positive constants $C$ and $\alpha$ independent of $k, \phi, \psi$ and $m$ such that

$$
\begin{aligned}
& \int_{a_{m}^{k}}^{a_{m}^{k+1}}\left|\widehat{\sigma}_{t, m, h}(\xi, \eta)\right|^{2} \frac{d t}{t} \leq C(m+1), \\
& \int_{a_{m}^{k}}^{a_{m}^{k+1}}\left|\widehat{\sigma}_{t, m, h}(\xi, \eta)\right|^{2} \frac{d t}{t} \leq C(m+1)\left|\phi\left(a_{m}^{k-1}\right) \xi\right|^{-\alpha /(m+1)}, \\
& \int_{a_{m}^{k}}^{a_{m}^{k+1}}\left|\widehat{\sigma}_{t, m, h}(\xi, \eta)\right|^{2} \frac{d t}{t} \leq C(m+1)\left|\phi\left(a_{m}^{k+1}\right) \xi\right|^{\alpha /(m+1)} .
\end{aligned}
$$

Proof. It is easy to see that (3.1) holds. Next, we prove (3.2). By a change of variable and Hölder's inequality we have

$$
\begin{aligned}
\left|\widehat{\sigma}_{t, m, h}(\xi, \eta)\right| & \leq \int_{t / 2}^{t}|h(s)|\left|\int_{\mathbb{S}^{n-1}} \Omega_{m}(x) e^{-i(\phi(s) \xi \cdot x+\eta \psi(s))} d \sigma(x)\right| \frac{d s}{s} \\
& \leq C\left(\int_{1 / 2}^{1}\left|\int_{\mathbb{S}^{n-1}} \Omega_{m}(x) e^{-i(\phi(s t) \xi \cdot x+\eta \psi(s t))} d \sigma(x)\right|^{\gamma^{\prime}} \frac{d s}{s}\right)^{1 / \gamma^{\prime}} .
\end{aligned}
$$

If $1<\gamma \leq 2$, by noticing that

we obtain

$$
\left|\int_{\mathbb{S}^{n-1}} \Omega_{m}(x) e^{-i(\phi(s t) \xi \cdot x+\eta \psi(s t))} d \sigma(x)\right| \leq 1
$$

$$
\left|\widehat{\sigma}_{t, m, h}(\xi, \eta)\right| \leq C\left(\int_{1 / 2}^{1}\left|\int_{\mathbb{S}^{n-1}} \Omega_{m}(x) e^{-i(\phi(s t) \xi \cdot x+\eta \psi(s t))} d \sigma(x)\right|^{2} \frac{d s}{s}\right)^{1 / \gamma^{\prime}} .
$$

Now, if $\gamma>2$, by Hölder's inequality, we get

$$
\begin{aligned}
\left|\widehat{\sigma}_{t, m, h}(\xi, \eta)\right| \leq & \left(\int_{t / 2}^{t}|h(s)|^{2} \frac{d s}{s}\right)^{1 / 2} \\
& \times\left(\int_{1 / 2}^{1}\left|\int_{\mathbb{S}^{n-1}} \Omega_{m}(x) e^{-i(\phi(s t) \xi \cdot x+\eta \psi(s t))} d \sigma(x)\right|^{2} \frac{d s}{s}\right)^{1 / 2}
\end{aligned}
$$




$$
\leq C\left(\int_{1 / 2}^{1}\left|\int_{\mathbb{S}^{n-1}} \Omega_{m}(x) e^{-i(\phi(s t) \xi \cdot x+\eta \psi(s t))} d \sigma(x)\right|^{2} \frac{d s}{s}\right)^{1 / 2} .
$$

So in either case, we get

$$
\left|\widehat{\sigma}_{t, m, h}(\xi, \eta)\right| \leq C\left(\int_{1 / 2}^{1}\left|\int_{\mathbb{S}^{n-1}} \Omega_{m}(x) e^{-i(\phi(s t) \xi \cdot x+\eta \psi(s t))} d \sigma(x)\right|^{2} \frac{d s}{s}\right)^{1 / \max \left\{\gamma^{\prime}, 2\right\}},
$$

which in turn implies that

$$
\left|\widehat{\sigma}_{t, m, h}(\xi, \eta)\right| \leq C\left(\int_{\mathbb{S}^{n-1} \times \mathbb{S}^{n-1}} \Omega_{m}(x) \overline{\Omega_{m}(y)} A_{t}(\xi, x, y) d \sigma(x) d \sigma(y)\right)^{1 / \max \left\{\gamma^{\prime}, 2\right\}}
$$

where

$$
A_{t}(\xi, x, y)=\int_{1 / 2}^{1} e^{-i \phi(s t) \xi \cdot(x-y)} \frac{d s}{s}
$$

Now

$$
A_{t}(\xi, x, y)=\int_{1 / 2}^{1} L_{t}^{\prime}(s) \frac{d s}{s}
$$

where

$$
L_{t}(s)=\int_{1 / 2}^{s} e^{-i \phi(t w) \xi \cdot(x-y)} d w, \quad 1 / 2 \leq s \leq 1 .
$$

By the mean value theorem and the assumptions on $\phi$ we have

$$
\frac{d}{d w}(\phi(t w))=t \phi^{\prime}(t w) \geq \frac{\phi(t w)}{w} \geq \frac{\phi(t / 2)}{s} \quad \text { for } 1 / 2 \leq w \leq s \leq 1 .
$$

By the last estimate and van der Corput's lemma,

$$
\left|L_{t}(s)\right| \leq\left|\frac{\phi(t / 2) \xi}{s}\right|^{-1}\left|\xi^{\prime} \cdot(x-y)\right|^{-1} .
$$

Thus, by integration by parts, we get

$$
\left|A_{t}(\xi, x, y)\right| \leq C|\phi(t / 2) \xi|^{-1}\left|\xi^{\prime} \cdot(x-y)\right|^{-1},
$$

which when combined with the trivial estimate $\left|A_{t}(\xi, x, y)\right| \leq \log 2$ and choosing $\alpha$ so that $0<2 \alpha<1$ yields

$$
\left|A_{t}(\xi, x, y)\right| \leq|\phi(t / 2) \xi|^{-\alpha}\left|\xi^{\prime} \cdot(x-y)\right|^{-\alpha} .
$$

By Hölder's inequality, (ii) and the choice of $\alpha$ we get

$$
\begin{aligned}
\left|\widehat{\sigma}_{t, m, h}(\xi, \eta)\right| \leq & C|\phi(t / 2) \xi|^{-\alpha / \max \left\{\gamma^{\prime}, 2\right\}}\left\|\Omega_{m}\right\|_{2}^{2 / \max \left\{\gamma^{\prime}, 2\right\}} \\
& \times\left(\int_{\mathbb{S}^{n-1} \times \mathbb{S}^{n-1}}\left|\xi^{\prime} \cdot(x-y)\right|^{-2 \alpha} d \sigma(x) d \sigma(y)\right)^{1 / \max \left\{\gamma^{\prime}, 2\right\}} \\
\leq & C|\phi(t / 2) \xi|^{-\alpha / \max \left\{\gamma^{\prime}, 2\right\}} a_{m}^{2 / \max \left\{\gamma^{\prime}, 2\right\}}
\end{aligned}
$$


and hence

$$
\int_{a_{m}^{k}}^{a_{m}^{k+1}}\left|\widehat{\sigma}_{t, m, h}(\xi, \eta)\right|^{2} \frac{d t}{t} \leq C(m+1)\left|\phi\left(\frac{1}{2} a_{m}^{k}\right) \xi\right|^{-2 \alpha / \max \left\{\gamma^{\prime}, 2\right\}} a_{m}^{4 / \max \left\{\gamma^{\prime}, 2\right\}},
$$

which when combined with the trivial estimate in (3.1) yields (3.2).

For (3.3), we use the cancellation property of $\Omega_{m}$ and the increasing property of $\phi$ to get

$$
\begin{aligned}
\left|\widehat{\sigma}_{t, m, h}(\xi, \eta)\right| & \leq \int_{t / 2}^{t}|h(s)|\left|\int_{\mathbb{S}^{n-1}} \Omega_{m}(x)\left(e^{-i(\phi(s) \xi \cdot x+\eta \psi(s))}-e^{-i \eta \psi(s)}\right) d \sigma(x)\right| \frac{d s}{s} \\
& \leq C|\xi \phi(t)|,
\end{aligned}
$$

which easily implies

$$
\int_{a_{m}^{k}}^{a_{m}^{k+1}}\left|\widehat{\sigma}_{t, m, h}(\xi, \eta)\right|^{2} \frac{d t}{t} \leq C(m+1)\left|\xi \phi\left(a_{m}^{k+1}\right)\right|^{2} .
$$

By combining the last estimate with (3.1) we get (3.3). This finishes the proof of Lemma 3.2.

By a similar argument we get

Lemma 3.3. Let $m \in \mathbb{N} \cup\{0\}$ and $\psi(\cdot)$ be an arbitrary function on $\mathbb{R}_{+}$. Let $\Omega_{m}(\cdot)$ and $\phi$ be as in Lemma 3.2. For $(\xi, \eta) \in \mathbb{R}^{n} \times \mathbb{R}$ let

$$
I_{m, k}(\xi, \eta)=\int_{a_{m}^{k}}^{a_{m}^{k+1}} \int_{1 / 2}^{1}\left|\int_{\mathbb{S}^{n-1}} \Omega_{m}(x) e^{-i(\phi(s t) \xi \cdot x+\eta \psi(s t))} d \sigma(x)\right|^{2} \frac{d s d t}{s t} .
$$

Then $\left|I_{m, k}(\xi, \eta)\right|$ satisfies the estimates in (3.1)-(3.3) (with the expression $\int_{a_{m}^{k}}^{a_{m}^{k+1}}\left|\widehat{\sigma}_{t, m, h}(\xi, \eta)\right|^{2} d t / t$ replaced by $\left.\left|I_{m, k}(\xi, \eta)\right|\right)$ for some positive constants $C$ and $\alpha$ independent of $k, m, \xi, \eta, \phi(\cdot)$ and $\psi(\cdot)$.

We shall need the following result from [AP1] which has its roots in [DR] and [FP1].

Lemma 3.4. Let $\left\{\sigma_{k}: k \in \mathbb{Z}\right\}$ be a sequence of Borel measures on $\mathbb{R}^{n}$. Let $L: \mathbb{R}^{n} \rightarrow \mathbb{R}^{m}$ be a linear transformation. Suppose that for all $k \in \mathbb{Z}$ and $\xi \in \mathbb{R}^{n}$, and some $a \geq 2, \alpha, C>0, A>1$ and $p_{0} \in(2, \infty)$, we have:

(i) $\left|\widehat{\sigma}_{k}(\xi)\right| \leq C A\left(a^{k A}|L(\xi)|\right)^{ \pm \alpha / A}$;

(ii) $\left\|\left(\sum_{k \in \mathbb{Z}}\left|\sigma_{k} * g_{k}\right|^{2}\right)^{1 / 2}\right\|_{p_{0}} \leq C A\left\|\left(\sum_{k \in \mathbb{Z}}\left|g_{k}\right|^{2}\right)^{1 / 2}\right\|_{p_{0}}$ for arbitrary functions $g_{k}$ on $\mathbb{R}^{n}$.

Then for $p_{0}^{\prime}<p<p_{0}$, there exists a positive constant $C_{p}$ such that

$$
\left\|\left(\sum_{k \in \mathbb{Z}}\left|\sigma_{k} * f\right|^{2}\right)^{1 / 2}\right\|_{L^{p}\left(\mathbb{R}^{n}\right)} \leq C_{p} A\|f\|_{L^{p}\left(\mathbb{R}^{n}\right)}
$$


for all $f$ in $L^{p}\left(\mathbb{R}^{n}\right)$. The constant $C_{p}$ is independent of $A$ and of the linear transformation $L$.

Now, we need to introduce two more maximal functions. First, define

$$
\lambda_{m, h}^{*}(f)=\sup _{k \in \mathbb{Z}} \int_{a_{m}^{k}}^{a_{m}^{k+1}}\left(\mu_{t, m, h} * f\right) \frac{d t}{t},
$$

where $f \geq 0, m \in \mathbb{N} \cup\{0\}$, and $\mu_{t, m, h}=\left|\sigma_{t, m, h}\right|$. We notice that if we define the measure $\lambda_{m, k, h}$ by

$$
\widehat{\lambda}_{m, k, h}(\xi, \eta)=\int_{a_{m}^{k}}^{a_{m}^{k+1}} \widehat{\mu}_{m, t, h}(\xi, \eta) \frac{d t}{t} \quad \text { for }(\xi, \eta) \in \mathbb{R}^{n} \times \mathbb{R},
$$

it is easy to see that

$$
\lambda_{m, h}^{*} f\left(x, x_{n+1}\right)=\sup _{k \in \mathbb{Z}}\left|\lambda_{m, k, h} * f\left(x, x_{n+1}\right)\right| .
$$

Lemma 3.5. Let $m \in \mathbb{N} \cup\{0\}$ and $h \in L^{\infty}\left(\mathbb{R}^{n}\right)$. Let $\Omega_{m}$ be a function on $\mathbb{S}^{n-1}$ satisfying conditions (i) and (ii) of Lemma 3.2. Assume that $\phi$ and $\psi$ are $C^{2}([0, \infty))$, convex, increasing functions with $\phi(0)=\psi(0)=0$. Then for every $1<p<\infty$, there exists a positive constant $C_{p}$ independent of $m$ such that

$$
\left\|\lambda_{m}^{*}(f)\right\|_{p} \leq C_{p}(m+1)\|f\|_{p}
$$

for every $f \in L^{p}\left(\mathbb{R}^{n+1}\right)$.

Proof. Fix a $\varphi \in \mathcal{S}\left(\mathbb{R}^{n}\right)$ such that $\widehat{\varphi}(\xi)=1$ for $|\xi| \leq 1$ and $\widehat{\varphi}(\xi)=0$ for $|\xi| \geq 2$. For each $t \in \mathbb{R}_{+}$, let $\left(\varphi_{t}\right)^{\wedge}(\xi)=\widehat{\varphi}(t \xi)$. Define the family of measures $\left\{\Upsilon_{m, t}\right\}_{t \in \mathbb{R}_{+}}$and $\left\{\vartheta_{m, k}\right\}_{k \in \mathbb{Z}}$ by

$$
\begin{aligned}
& \widehat{\Upsilon}_{m, t}(\xi, \eta)=\widehat{\mu}_{m, t, h}(\xi, \eta)-\widehat{\mu}_{m, t, h}(0, \eta)\left(\varphi_{t}\right)^{\wedge}(\xi), \\
& \widehat{\vartheta}_{m, k}(\xi, \eta)=\int_{a_{m}^{k}}^{a_{m}^{k+1}} \widehat{\Upsilon}_{m, t}(\xi, \eta) \frac{d t}{t} .
\end{aligned}
$$

Now, let

$$
\begin{aligned}
g_{m}(f) & =\left(\sum_{k \in \mathbb{Z}}\left|\vartheta_{m, k} * f\right|^{2}\right)^{1 / 2}, \quad \vartheta_{m}^{*}(f)=\sup _{k \in \mathbb{Z}}|| \vartheta_{m, k}|* f|, \\
M_{\phi} f\left(x, x_{n+1}\right) & =\sup _{t \in \mathbb{R}_{+}}\left|\int_{t / 2}^{t} f\left(x, x_{n+1}-\phi(s)\right) \frac{d s}{s}\right| .
\end{aligned}
$$

By the proof of Lemma 3.2 and the choice of $\varphi$ we find that $\vartheta_{m, k}$ satisfies 
the estimates

$$
\begin{aligned}
\left\|\vartheta_{m, k}\right\| & \leq C(m+1), \\
\left|\widehat{\vartheta}_{m, k}(\xi, \eta)\right| & \leq C(m+1)\left|\phi\left(a_{m}^{k-1}\right) \xi\right|^{-\alpha /(m+1)}, \\
\left|\widehat{\vartheta}_{m, k}(\xi, \eta)\right| & \leq C(m+1)\left|\phi\left(a_{m}^{k+1}\right) \xi\right|^{\alpha /(m+1)} .
\end{aligned}
$$

By (3.5) we have

$$
\begin{aligned}
& \vartheta_{m}^{*} f\left(x, x_{n+1}\right) \\
& \quad \leq g_{m} f\left(x, x_{n+1}\right)+C(m+1)\left(\left(\mathcal{M}_{\mathbb{R}^{n}} \otimes \mathrm{id}_{\mathbb{R}^{1}}\right) \circ M_{\phi}\right) f\left(x, x_{n+1}\right), \\
& \lambda_{m}^{*} f\left(x, x_{n+1}\right) \\
& \quad \leq g_{m} f\left(x, x_{n+1}\right)+2 C(m+1)\left(\left(\mathcal{M}_{\mathbb{R}^{n}} \otimes \mathrm{id}_{\mathbb{R}^{1}}\right) \circ M_{\phi}\right) f\left(x, x_{n+1}\right),
\end{aligned}
$$

where $\mathcal{M}_{\mathbb{R}^{d}}$ is the classical Hardy-Littlewood maximal function on $\mathbb{R}^{d}$. By the argument in [DR, p. 558] we get

$$
M_{\phi} f\left(x, x_{n+1}\right) \leq C \mathcal{M}_{\mathbb{R}^{1}} f(x, \cdot)\left(x_{n+1}\right) .
$$

By (3.6)-(3.8) and Plancherel's theorem,

$$
\left\|g_{m}(f)\right\|_{2} \leq C(m+1)\|f\|_{2} .
$$

By the boundedness of $\mathcal{M}_{\mathbb{R}^{n}}$ on $L^{p}(1<p<\infty)$, (3.9) and (3.11)-(3.12) we get

$$
\left\|\vartheta_{m}^{*}(f)\right\|_{2} \leq C(m+1)\|f\|_{2} .
$$

Now, by (3.6), (3.13) and applying the proof of the lemma in [DR, p. 544] with $p_{0}=4$ and $q=2$, we obtain

$$
\left\|\left(\sum_{k \in \mathbb{Z}}\left|\vartheta_{m, k} * g_{k}\right|^{2}\right)^{1 / 2}\right\|_{4} \leq C(m+1)\left\|\left(\sum_{k \in \mathbb{Z}}\left|g_{k}\right|^{2}\right)^{1 / 2}\right\|_{4}
$$

for arbitrary functions $\left\{g_{k}\right\}_{k \in \mathbb{Z}}$ on $\mathbb{R}^{n+1}$. By (3.6)-(3.8), (3.14) and applying Lemma 3.4 we get

$$
\left\|g_{m}(f)\right\|_{p} \leq C_{p}(m+1)\|f\|_{p}
$$

for all $p \in(4 / 3,4)$ and $f \in L^{p}\left(\mathbb{R}^{n+1}\right)$. By replacing $p=2$ with $p=4 / 3+\varepsilon$ with $\varepsilon \rightarrow 0^{+}$in (3.12) and repeating the preceding arguments, we get (3.15) for every $p \in(8 / 7,8)$ and $f \in L^{p}\left(\mathbb{R}^{n+1}\right)$. By continuing this process we ultimately get

$$
\left\|g_{m}(f)\right\|_{p} \leq C_{p}(m+1)\|f\|_{p}
$$

for all $p \in(1, \infty)$ and $f \in L^{p}\left(\mathbb{R}^{n+1}\right)$. Therefore, by (3.10) and (3.16), we obtain (3.4), which completes the proof of the lemma.

Lemma 3.6. Let $m, \Omega_{m}, \phi$ and $\psi$ be as in Lemma 3.5. Then for every $1<p<\infty$, there exists a positive constant $C_{p}$ independent of $m$ such that

$$
\left\|F_{m}^{*}(f)\right\|_{p} \leq C_{p}(m+1)\|f\|_{p}
$$


for every $f \in L^{p}\left(\mathbb{R}^{n+1}\right)$, where

$F_{m}^{*} f\left(x, x_{n+1}\right)$

$$
=\sup _{k \in \mathbb{Z}}\left(\int_{a_{m}^{k}<|u| \leq a_{m}^{k+1}} \int_{1 / 2}^{1} f\left(x-\phi(|s u|) u^{\prime}, x_{n+1}-\psi(|s u|)\right) \frac{\Omega\left(u^{\prime}\right)}{|u|^{n}} \frac{d s}{s} d u\right) .
$$

This lemma can be proved by using Lemmas 3.3 and 3.4 and following an argument similar to the one in the proof of Lemma 3.5. The details will be omitted.

Lemma 3.7. Let $h \in \Delta_{\gamma}\left(\mathbb{R}_{+}\right)$for some $\gamma>1$ and let $\Omega_{m}, \phi$ and $\psi$ be as in Lemma 3.2. Then for $\gamma^{\prime}<p \leq \infty$, there exists a positive constant $C_{p}$ independent of $m$ such that

$$
\left\|\sigma_{m, h}^{*}(f)\right\|_{p} \leq C_{p}(m+1)^{1 / \gamma^{\prime}}\|f\|_{p}
$$

for all $f \in L^{p}\left(\mathbb{R}^{n+1}\right)$.

Proof. By using Hölder's inequality we have $\sigma_{m, h}^{*}(f) \leq C\left(\Upsilon_{m}^{*}\left(|f|^{\gamma^{\prime}}\right)\right)^{1 / \gamma^{\prime}}$, where

$$
\int_{\mathbb{R}^{n+1}} f d \Upsilon_{t, m}=\int_{t / 2<|u| \leq t} f\left(\phi(|u|) u^{\prime}, \psi(|u|)\right)|u|^{-n} \Omega_{m}\left(u^{\prime}\right) d u
$$

and $\Upsilon_{m}^{*}(f)=\sup _{t \in \mathbb{R}_{+}}|| \Upsilon_{t, m}|* f|$. Therefore, to prove (3.18) it suffices to show that

$$
\left\|\Upsilon_{m}^{*}(f)\right\|_{L^{p}\left(\mathbb{R}^{n+1}\right)} \leq C_{p}(m+1)\|f\|_{L^{p}\left(\mathbb{R}^{n+1}\right)} \quad \text { for } 1<p \leq \infty .
$$

It is easy to see that

$$
\begin{aligned}
& \Upsilon_{m}^{*} f\left(x, x_{n+1}\right) \\
& \leq 2 \sup _{k \in \mathbb{Z}}\left(\int_{a_{m}^{k}<|u| \leq a_{m}^{k+1}}\left|f\left(x-\phi(|u|) u^{\prime}, x_{n+1}-\psi(|u|)\right)\right| \frac{\left|\Omega_{m}\left(u^{\prime}\right)\right|}{|u|^{n}} d u\right),
\end{aligned}
$$

and it now suffices to adapt the argument in the proof of Lemma 3.5.

Lemma 3.8. Let $h \in \Delta_{\gamma}\left(\mathbb{R}_{+}\right)$for some $\gamma \geq 2$ and $\gamma^{\prime}<p<\infty$. Also, let $m, \Omega_{m}, \phi$ and $\psi$ be as in Lemma 3.5. Then there exists a positive constant $C_{p}$ such that

$$
\left\|\left(\sum_{k \in \mathbb{Z}} \int_{a_{m}^{k}}^{a_{m}^{k+1}}\left|\sigma_{t, m, h} * g_{k}\right|^{2} \frac{d t}{t}\right)^{1 / 2}\right\|_{p} \leq C_{p}(m+1)^{1 / \gamma^{\prime}}\left\|\left(\sum_{k \in \mathbb{Z}}\left|g_{k}\right|^{2}\right)^{1 / 2}\right\|_{p}
$$

for any sequence $\left\{g_{k}\right\}_{k \in \mathbb{Z}}$ of functions on $\mathbb{R}^{n+1}$. 
Proof. Let $\gamma^{\prime}<p<\infty$. By a change of variable, we have

$$
\left(\sum_{k \in \mathbb{Z}} \int_{a_{m}^{k}}^{a_{m}^{k+1}}\left|\sigma_{t, m, h} * g_{k}\right|^{2} \frac{d t}{t}\right)^{1 / 2} \leq\left(\sum_{k \in \mathbb{Z}} \int_{1}^{a_{m}}\left|\sigma_{a_{m}^{k} t, m, h} * g_{k}\right|^{2} \frac{d t}{t}\right)^{1 / 2} .
$$

By Hölder's inequality we get

$$
\begin{aligned}
& \left|\sigma_{t, m, h} * g_{k}\left(x, x_{n+1}\right)\right|^{\gamma^{\prime}} \\
& \leq C\left(\int_{a_{m}^{k} t / 2}^{a_{m}^{k} t} \int_{\mathbb{S}^{n-1}}\left|\Omega_{m}(y)\right|\left|g_{k}\left(x-\phi(s) u, x_{n+1}-\psi(s)\right)\right|^{\gamma^{\prime}} d \sigma(y) \frac{d s}{s}\right) .
\end{aligned}
$$

Let $d=p / \gamma^{\prime}$. By duality, there is a nonnegative function $f \in L^{d^{\prime}}\left(\mathbb{R}^{n+1}\right)$ satisfying $\|f\|_{L^{d^{\prime}}\left(\mathbb{R}^{n+1}\right)} \leq 1$ such that

$$
\begin{aligned}
& \left\|\left(\sum_{k \in \mathbb{Z}} \int_{1}^{a_{m}}\left|\sigma_{a_{m}^{k} t, m, h} * g_{k}\right| \gamma^{\prime} \frac{d t}{t}\right)^{1 / \gamma^{\prime}}\right\|_{L^{p}\left(\mathbb{R}^{n+1}\right)}^{\gamma^{\prime}} \\
& \quad=\int_{\mathbb{R}^{n}} \sum_{k \in \mathbb{Z}} \int_{1}^{a_{m}}\left|\sigma_{a_{m}^{k} t, m, h} * g_{k}\left(x, x_{n+1}\right)\right|^{\gamma^{\prime}} \frac{d t}{t} f\left(x, x_{n+1}\right) d x d x_{n+1} .
\end{aligned}
$$

Therefore, by (3.21) and a change of variable we get

$$
\begin{aligned}
& \left\|\left(\sum_{k \in \mathbb{Z}} \int_{1}^{a_{m}}\left|\sigma_{a_{m}^{k} t, m, h} * g_{k}\right|^{\gamma^{\prime}} \frac{d t}{t}\right)^{1 / \gamma^{\prime}}\right\|_{L^{p\left(\mathbb{R}^{n+1}\right)}}^{\gamma^{\prime}} \\
& \leq C \int_{\mathbb{R}^{n+1}} \sum_{k \in \mathbb{Z}}\left|g_{k}\left(x, x_{n+1}\right)\right|^{\gamma^{\prime}} \lambda_{m, 1}^{*} \widetilde{f}\left(-x,-x_{n+1}\right) d x d x_{n+1},
\end{aligned}
$$

where $\widetilde{f}\left(x, x_{n+1}\right)=f\left(-x,-x_{n+1}\right)$. By Hölder's inequality, we obtain

$$
\begin{aligned}
\left\|\left(\sum_{k \in \mathbb{Z}} \int_{1}^{a_{m}}\left|\sigma_{a_{m}^{k} t, m, h} * g_{k}\right|^{\gamma^{\prime}} \frac{d t}{t}\right)^{1 / \gamma^{\prime}}\right\|_{L^{p}\left(\mathbb{R}^{n+1}\right)}^{\gamma^{\prime}} \\
\leq C\left\|\left(\sum_{k \in \mathbb{Z}}\left|g_{k}\right|^{\gamma^{\prime}}\right)^{1 / \gamma^{\prime}}\right\|_{L^{p}\left(\mathbb{R}^{n+1}\right)}^{\gamma^{\prime}}\left\|\lambda_{m, 1}^{*} \widetilde{f}\right\|_{L^{d^{\prime}}\left(\mathbb{R}^{n+1}\right)} .
\end{aligned}
$$

By Lemma 3.5, we have

$$
\begin{aligned}
\left\|\left(\sum_{k \in \mathbb{Z}} \int_{1}^{a_{m}}\left|\sigma_{a_{m}^{k} t, m, h} * g_{k}\right|^{\gamma^{\prime}} \frac{d t}{t}\right)^{1 / \gamma^{\prime}}\right\|_{L^{p}\left(\mathbb{R}^{n+1}\right)} \\
\leq C(m+1)^{1 / \gamma^{\prime}}\left\|\left(\sum_{k \in \mathbb{Z}}\left|g_{k}\right|^{\gamma^{\prime}}\right)^{1 / \gamma^{\prime}}\right\|_{L^{p}\left(\mathbb{R}^{n+1}\right)} .
\end{aligned}
$$


On the other hand, by Lemma 3.7, we get

$$
\begin{aligned}
& \left\|\sup _{k \in \mathbb{Z}} \sup _{t \in\left[1, a_{m}\right]}\left|\sigma_{a_{m}^{k} t, m, h} * g_{k}\right|\right\|_{L^{p}\left(\mathbb{R}^{n+1}\right)} \\
& \leq\left\|\sigma_{m, h}^{*}\left(\sup _{k \in \mathbb{Z}}\left|g_{k}\right|\right)\right\|_{L^{p}\left(\mathbb{R}^{n+1}\right)} \leq C(m+1)^{1 / \gamma^{\prime}}\left\|\sup _{k \in \mathbb{Z}}\left|g_{k}\right|\right\|_{L^{p}\left(\mathbb{R}^{n+1}\right)}
\end{aligned}
$$

Define the linear operator $S$ on any function $g=g_{k}\left(x, x_{n+1}\right)$ by

$$
S g_{k}\left(x, x_{n+1}\right)=\sigma_{a_{m}^{k} t, h} * g_{k}\left(x, x_{n+1}\right) .
$$

Then by (3.24), we have

$$
\begin{aligned}
& \text { \| \| \|S } S(g)\left\|_{L^{\gamma^{\prime}\left(\left[1, a_{m}\right], d t / t\right)}}\right\|_{l^{\gamma^{\prime}(\mathbb{Z})}} \|_{L^{p}\left(\mathbb{R}^{n+1}\right)} \\
& \leq C(m+1)^{1 / \gamma^{\prime}}\|\| g\left\|_{l^{\gamma^{\prime}(\mathbb{Z})}}\right\|_{L^{p}\left(\mathbb{R}^{n+1}\right)} .
\end{aligned}
$$

Also, by (3.25) we get

$$
\begin{aligned}
\|\|\|S(g)\|_{L^{\infty}\left(\left[1, a_{m}\right], d t / t\right)} \|_{l^{\infty}(\mathbb{Z})} & \|_{L^{p}\left(\mathbb{R}^{n+1}\right)} \\
& =\|\|\|S(g)\|_{L^{\infty}\left(\left[1, a_{m}\right], d t\right)}\left\|_{l^{\infty}(\mathbb{Z})}\right\|_{L^{p}\left(\mathbb{R}^{n+1}\right)} \\
& \leq C(m+1)^{1 / \gamma^{\prime}}\|\| g\left\|_{l^{\infty}(\mathbb{Z})}\right\|_{L^{p}\left(\mathbb{R}^{n+1}\right)}
\end{aligned}
$$

Therefore, we can interpolate (3.26) and (3.27) (see [GR, p. 481] for vectorvalued interpolation) to get (3.20). The lemma is proved.

Lemma 3.9. Let $h \in \Delta_{\gamma}\left(\mathbb{R}_{+}\right)$for some $1<\gamma \leq 2$ and $2 \leq p<$ $2 \gamma /(2-\gamma)$. Also, let $m, \Omega_{m}, \phi$ and $\psi$ be as in Lemma 3.5. Then there exists a positive constant $C_{p}$ such that

$$
\left\|\left(\sum_{k \in \mathbb{Z}} \int_{a_{m}^{k}}^{a_{m}^{k+1}}\left|\sigma_{t, m, h} * g_{k}\right|^{2} \frac{d t}{t}\right)^{1 / 2}\right\|_{p} \leq C_{p}(m+1)^{1 / 2}\left\|\left(\sum_{k \in \mathbb{Z}}\left|g_{k}\right|^{2}\right)^{1 / 2}\right\|_{p}
$$

for any sequence $\left\{g_{k}\right\}_{k \in \mathbb{Z}}$ of functions on $\mathbb{R}^{n+1}$.

Proof. We use an argument similar to the one in the proof of Theorem 7.5 in [FP1]. By duality there exists a nonnegative function $f \in L^{(p / 2)^{\prime}}\left(\mathbb{R}^{n+1}\right)$ with $\|f\|_{(p / 2)^{\prime}} \leq 1$ such that

$$
\begin{aligned}
& \left\|\left(\sum_{k \in \mathbb{Z}} \int_{a_{m}^{k}}^{a_{m}^{k+1}}\left|\sigma_{t, m, h} * g_{k}\right|^{2} \frac{d t}{t}\right)^{1 / 2}\right\|_{p} \\
& \quad=\sum_{k \in \mathbb{Z} \mathbb{R}^{n+1}} \int_{a_{m}^{k}}^{a_{m}^{k+1}}\left|\sigma_{t, m, h} * g_{k}\left(x, x_{n+1}\right)\right|^{2} \frac{d t}{t} f\left(x, x_{n+1}\right) d x d x_{n+1} .
\end{aligned}
$$

By Schwarz's inequality we get 


$$
\begin{aligned}
& \left|\sigma_{t, m, h} * g_{k}\left(x, x_{n+1}\right)\right|^{2} \\
& \quad \leq C\left(\int_{t / 2}^{t} \int_{\mathbb{S}^{n-1}}\left|g_{k}\left(x-\phi(s) u, x_{n+1}-\psi(s)\right)\right|^{2}\left|\Omega_{m}(y)\right||h(s)|^{2-\gamma} d \sigma(y) \frac{d s}{s}\right) .
\end{aligned}
$$

Therefore, by a change of variable we have

$$
\begin{aligned}
& \left\|\left(\sum_{k \in \mathbb{Z}} \int_{a_{m}^{k}}^{a_{m}^{k+1}}\left|\sigma_{t, m, h} * g_{k}\right|^{2} \frac{d t}{t}\right)^{1 / 2}\right\|_{L^{p}\left(\mathbb{R}^{n+1}\right)}^{2} \\
& \quad \leq C \int_{\mathbb{R}^{n+1}}\left(\sum_{k \in \mathbb{Z}}\left|g_{k}\left(x, x_{n+1}\right)\right|^{2}\right) \lambda_{m,|h|^{2-\gamma}}^{*} \tilde{f}\left(-x,-x_{n+1}\right) d x d x_{n+1} .
\end{aligned}
$$

By Lemma 3.5 and noticing that $|h(\cdot)|^{2-\gamma} \in \Delta_{\gamma /(2-\gamma)}\left(\mathbb{R}_{+}\right)$and $(p / 2)^{\prime}>$ $(\gamma /(2-\gamma))^{\prime}$ we obtain

(3.30) $\left\|\lambda_{m,|h|^{2-\gamma}}^{*}(f)\right\|_{L^{(p / 2)^{\prime}\left(\mathbb{R}^{n+1}\right)}} \leq C_{p}(m+1)\|f\|_{L^{(p / 2)^{\prime}}\left(\mathbb{R}^{n+1}\right)} \leq C_{p}(m+1)$.

Thus, by (3.29)-(3.30) and Hölder's inequality we get (3.28) for $2 \leq p<$ $2 \gamma /(2-\gamma)$.

LEMMA 3.10. Let $m \in \mathbb{N} \cup\{0\}$ and $h \in \Delta_{\gamma}\left(\mathbb{R}_{+}\right)$for some $1<\gamma \leq 2$ and $2 \gamma /(3 \gamma-2)<p<2$. Also, let $m, \Omega_{m}$, $\phi$ and $\psi$ be as in Lemma 3.5. Then there exists a positive constant $C_{p}$ such that

$$
\begin{aligned}
\|\left(\sum_{k \in \mathbb{Z}} \int_{a_{m}^{k}}^{a_{m}^{k+1}}\left|\sigma_{t, m, h} * g_{k}\right|^{2}\right. & \left.\frac{d t}{t}\right)^{1 / 2} \|_{p} \\
\leq & C_{p}(m+1)^{(3 \gamma-2) /(2 \gamma)}\left\|\left(\sum_{k \in \mathbb{Z}}\left|g_{k}\right|^{2}\right)^{1 / 2}\right\|_{p}
\end{aligned}
$$

for any sequence $\left\{g_{k}\right\}_{k \in \mathbb{Z}}$ of functions on $\mathbb{R}^{n+1}$.

Proof. Assume $2 \gamma /(3 \gamma-2)<p<2$. By a duality argument, there exist functions $f=f_{k}\left(x, x_{n+1}, t\right)=f_{k, t}\left(x, x_{n+1}\right)$ defined on $\mathbb{R}^{n+1} \times \mathbb{R}^{+}$with \|\|$\left\|f_{k, t}(\cdot, \cdot)\right\|_{L^{2}\left(\left[a_{m}^{k}, a_{m}^{k+1}\right], d t / t\right)}\left\|_{l^{2}}\right\|_{L^{p^{\prime}}} \leq 1$ such that

$$
\begin{gathered}
\left\|\left(\sum_{k \in \mathbb{Z}} \int_{a_{m}^{k}}^{a_{m}^{k+1}}\left|\sigma_{t, m, h} * g_{k}\right|^{2} \frac{d t}{t}\right)^{1 / 2}\right\|_{p} \\
\quad=\int_{\mathbb{R}^{n+1}} \sum_{k \in \mathbb{Z}} \int_{a_{m}^{k}}^{a_{m}^{k+1}}\left(\sigma_{t, m, h} * g_{k}\left(x, x_{n+1}\right)\right) f_{k, t}\left(x, x_{n+1}\right) \frac{d t}{t} d x d x_{n+1} \\
\leq C_{p}(m+1)^{1 / 2}\left\|\left(\sum_{k \in \mathbb{Z}}\left|g_{k}\right|^{2}\right)^{1 / 2}\right\|_{p}\left\|(T(f))^{1 / 2}\right\|_{p^{\prime}},
\end{gathered}
$$


where

$$
T f\left(x, x_{n+1}\right)=\sum_{k \in \mathbb{Z}} \int_{a_{m}^{k}}^{a_{m}^{k+1}}\left|\sigma_{t, m, h} * f_{k, t}\left(x, x_{n+1}\right)\right|^{2} \frac{d t}{t} .
$$

Now, since $p^{\prime}>2$, there exists $H \in L^{\left(p^{\prime} / 2\right)^{\prime}}\left(\mathbb{R}^{n+1}\right)$ with $\|H\|_{p^{\prime} / 2} \leq 1$ such that

$$
\|T(f)\|_{p^{\prime} / 2}=\sum_{k \in \mathbb{Z}} \int_{\mathbb{R}^{n+1}} \int_{a_{m}^{k}}^{a_{m}^{k+1}}\left|\sigma_{t, m, h} * f_{k, t}\left(x, x_{n+1}\right)\right|^{2} H\left(x, x_{n+1}\right) \frac{d t}{t} d x d x_{n+1} .
$$

By an argument similar to the one in the proof of Lemma 3.9, we have $\|T(f)\|_{p^{\prime} / 2}$

$$
\begin{aligned}
& \leq C \int_{\mathbb{R}^{n+1}} \sigma_{m,|h|^{2-\gamma}}^{*} \tilde{H}\left(-x,-x_{n+1}\right)\left(\sum_{k \in \mathbb{Z}} \int_{a_{m}^{k}}^{a_{m}^{k+1}}\left|f_{k, t}\left(x, x_{n+1}\right)\right|^{2} \frac{d t}{t}\right) d x d x_{n+1} \\
& \leq C\left\|\left(\sum_{k \in \mathbb{Z}} \int_{E_{\mu, k}}\left|f_{k, t}(\cdot, \cdot)\right|^{2} \frac{d t}{t}\right)\right\|_{p^{\prime} / 2}\left\|\sigma_{m,|h|^{2-\gamma}}^{*}(\tilde{H})\right\|_{\left(p^{\prime} / 2\right)^{\prime}} .
\end{aligned}
$$

By invoking Lemma 3.7 and Hölder's inequality we obtain

$$
\left\|\sigma_{m,|h|^{2-\gamma}}^{*}(\widetilde{H})\right\|_{\left(p^{\prime} / 2\right)^{\prime}} \leq C_{p}(m+1)^{2 / \gamma^{\prime}}\|H\|_{\left(p^{\prime} / 2\right)^{\prime}} \leq C_{p}(m+1)^{2 / \gamma^{\prime}} .
$$

Thus by our choice of $f_{k, t}\left(x, x_{n+1}\right)$,

$$
\|T(f)\|_{p^{\prime} / 2} \leq C_{p}\left\|\left(\sum_{k \in \mathbb{Z}} \int_{a_{m}^{k}}^{a_{m}^{k+1}}\left|f_{k, t}(\cdot, \cdot)\right|^{2} \frac{d t}{t}\right)\right\|_{p^{\prime} / 2} \leq C_{p}(m+1)^{2 / \gamma^{\prime}},
$$

which along with (3.32) gives (3.31) for $2 \gamma /(3 \gamma-2)<p<2$. The proof is complete.

LEMMA 3.11. Let $m \in \mathbb{N} \cup\{0\}$ and $h \in \Delta_{\gamma}\left(\mathbb{R}_{+}\right)$for some $1<\gamma \leq 2$ and $2 \gamma /(2 \gamma-1)<p<2$. Also, let $m, \Omega_{m}$, $\phi$ and $\psi$ be as in Lemma 3.5. Then there exists a positive constant $C_{p}$ such that

$$
\begin{aligned}
\|\left(\sum_{k \in \mathbb{Z}} \int_{a_{m}^{k}}^{a_{m}^{k+1}}\left|\sigma_{t, m, h} * g_{k}\right|^{2}\right. & \left.\frac{d t}{t}\right)^{1 / 2} \|_{p} \\
\leq & C_{p}(m+1)^{(2 \gamma-1) /(2 \gamma)}\left\|\left(\sum_{k \in \mathbb{Z}}\left|g_{k}\right|^{2}\right)^{1 / 2}\right\|_{p}
\end{aligned}
$$

for any sequence $\left\{g_{k}\right\}_{k \in \mathbb{Z}}$ of functions on $\mathbb{R}^{n+1}$.

Proof. We adopt the same notations as in the proof of Lemma 3.8. Since $\left\|\sigma_{t, m, h}\right\| \leq C$ we get 


$$
\begin{aligned}
\| & \|S(g)\|_{L^{1}\left(\left[1, a_{m}\right], d t / t\right)}\left\|_{l^{1}(\mathbb{Z})}\right\|_{L^{1}\left(\mathbb{R}^{n+1}\right)} \\
& =\int_{\mathbb{R}^{n+1}} \sum_{k \in \mathbb{Z}} \int_{1}^{a_{m}}\left|\sigma_{t, m, h} * g_{k}\left(x, x_{n+1}\right)\right| \frac{d t}{t} d x d x_{n+1} \\
& =\sum_{k \in \mathbb{Z}} \int_{1}^{a_{m}}\left\|\sigma_{t, m, h} * g_{k}\right\|_{L^{1}\left(\mathbb{R}^{n+1}\right)} \frac{d t}{t} \\
& \leq \sum_{k \in \mathbb{Z}} \int_{1}^{a_{m}}\left\|\sigma_{t, m, h}\right\|\left\|g_{k}\right\|_{L^{1}\left(\mathbb{R}^{n+1}\right)} \frac{d t}{t} \leq C(m+1)\left\|\sum_{k \in \mathbb{Z}}\left|g_{k}\right|\right\|_{L^{1}\left(\mathbb{R}^{n+1}\right)}
\end{aligned}
$$

By interpolating between (3.34) and (3.27) (see [GR, p. 481]) we get (3.33).

\section{Proof of main theorems}

Proof of Theorem 1.3(a). Assume that $\Omega$ satisfies (1.1) and belongs to $L(\log L)^{1 / \gamma^{\prime}}\left(\mathbb{S}^{n-1}\right)$ and $1 \leq \gamma \leq 2$. First, by Minkowski's inequality we have

$$
\begin{aligned}
\mathcal{M}_{\Omega, \phi, \psi, h}^{\varrho} f\left(x, x_{n+1}\right)= & \int_{0}^{\infty} \mid \sum_{k=0}^{\infty} \frac{1}{t^{\varrho}} \int_{2^{-k-1} t<|y| \leq 2^{-k} t} \frac{\Omega\left(u^{\prime}\right)}{|u|^{n-\varrho}} h(|u|) \\
& \left.\times\left. f\left(x-\phi(|u|) u^{\prime}, x_{n+1}-\psi(|u|)\right) d u\right|^{2} \frac{d t}{t}\right)^{1 / 2} \\
\leq & \sum_{k=0}^{\infty}\left(\int_{0}^{\infty} \mid \frac{1}{t^{\varrho}} \int_{2^{-k-1}} \int_{t<|y| \leq 2^{-k} t} \frac{\Omega\left(u^{\prime}\right)}{|u|^{n-\varrho}} h(|u|)\right. \\
& \times f\left(x-\phi(|u|) u^{\prime}, x_{n+1}-\left.\psi(|u|) d u\right|^{2} \frac{d t}{t}\right)^{1 / 2} \\
= & A_{\sigma} \widetilde{\mathcal{M}}_{\Omega, \phi, \psi, h}^{\varrho} f\left(x, x_{n+1}\right),
\end{aligned}
$$

where

$$
\begin{aligned}
\widetilde{\mathcal{M}}_{\Omega, \phi, \psi, h}^{\varrho} f\left(x, x_{n+1}\right)= & \left(\int_{0}^{\infty} \mid \frac{1}{t^{\varrho}} \int_{t / 2 t<|u| \leq t} \frac{\Omega\left(u^{\prime}\right)}{|u|^{n-\varrho}} h(|u|)\right. \\
& \left.\times\left. f\left(x-\phi(|u|) u^{\prime}, x_{n+1}-\psi(|u|)\right) d u\right|^{2} \frac{d t}{t}\right)^{1 / 2} .
\end{aligned}
$$

Let

$$
\widetilde{M}_{\phi, \psi, \Omega}^{(\gamma)} f\left(x, x_{n+1}\right)=\sup _{\|h\|_{L \gamma\left(\mathbb{R}_{+}, d t / t\right)} \leq 1}\left|\widetilde{\mathcal{M}}_{\Omega, \phi, \psi, h}^{\varrho} f\left(x, x_{n+1}\right)\right|,
$$

where the supremum is taken over all measurable radial functions $h$ with $\|h\|_{L^{\gamma}\left(\mathbb{R}_{+}, d t / t\right)} \leq 1$. Thus in view of $(4.1)$, we get

$$
M_{\phi, \psi, \Omega}^{(\gamma)} f\left(x, x_{n+1}\right) \leq C \widetilde{M}_{\phi, \psi, \Omega}^{(\gamma)} f\left(x, x_{n+1}\right)
$$


and hence here and in the following we shall deal with the maximal operator $\widetilde{M}_{\phi, \psi, \Omega}^{(\gamma)}$ instead of $M_{\phi, \psi, \Omega}^{(\gamma)}$. For convenience, we normalize $\sigma$ so that $\sigma\left(\mathbb{S}^{n-1}\right)=1$. Now, we decompose $\Omega$ as follows: For $m \in \mathbb{N}$, let $\mathbf{J}_{m}$ be the set of points $x \in \mathbb{S}^{n-1}$ which satisfy $2^{m} \leq|\Omega(x)|<2^{m+1}$. Also, we let $\mathbf{J}_{0}$ be the set of all $x \in \mathbb{S}^{n-1}$ which satisfy $|\Omega(x)|<2$. For $m \in \mathbb{N} \cup\{0\}$, set $b_{m}=\Omega \chi_{\mathbf{J}_{m}}$ and $C_{m}=\left\|b_{m}\right\|_{1}$. Set $I=\left\{m \in \mathbb{N}: C_{m} \geq 2^{-4 m}\right\}$ and define the sequence $\left\{\Omega_{m}\right\}_{m \in I \cup\{0\}}$ of functions by

$$
\begin{aligned}
& \Omega_{0}(x)=\sum_{m \in\{0\} \cup(\mathbb{N}-I)} b_{m}(x)-\sum_{m \in\{0\} \cup(\mathbb{N}-I)}\left(\int_{\mathbb{S}^{n-1}} b_{m}(x) d \sigma(x)\right), \\
& \Omega_{m}(x)=\left(C_{m}\right)^{-1}\left(b_{m}(x)-\int_{\mathbb{S}^{n-1}} b_{m}(x) d \sigma(x)\right) \quad \text { for } m \in I .
\end{aligned}
$$

It is easy to verify that for all $m \in I \cup\{0\}$ and some positive constant $C$,

$$
\begin{aligned}
& \left\|\Omega_{m}\right\|_{2} \leq C a_{m}^{2}, \quad\left\|\Omega_{m}\right\|_{1} \leq C, \\
& \sum_{m \in I \cup\{0\}}(m+1)^{1 / \gamma^{\prime}} C_{m} \leq C\|\Omega\|_{L(\log L)^{1 / \gamma^{\prime}\left(\mathbb{S}^{n-1}\right)}}, \\
& \int_{\mathbb{S}^{n-1}} \Omega_{m}(u) d \sigma(u)=0, \quad \Omega=\sum_{m \in I \cup\{0\}} C_{m} \Omega_{m} .
\end{aligned}
$$

By (4.4), we have

$$
\left\|\widetilde{M}_{\phi, \psi, \Omega}^{(\gamma)} f\right\|_{L^{p}\left(\mathbb{R}^{n+1}\right)} \leq \sum_{m \in I \cup\{0\}} C_{m}\left\|\widetilde{M}_{\phi, \psi, \Omega_{m}}^{(\gamma)} f\right\|_{L^{p}\left(\mathbb{R}^{n+1}\right)}
$$

and hence the proof of Theorem 1.3(a) is completed if we can show that

$$
\left\|\widetilde{M}_{\phi, \psi, \Omega_{m}}^{(\gamma)} f\right\|_{L^{p}\left(\mathbb{R}^{n+1}\right)} \leq C_{p}(m+1)^{1 / \gamma^{\prime}}\|f\|_{L^{p}\left(\mathbb{R}^{n+1}\right)}
$$

for all $m \in I \cup\{0\}$, and for $\gamma^{\prime} \leq p<\infty$ if $1<\gamma \leq 2$, and $p=\infty$ if $\gamma=1$. We will first handle the cases $\gamma=1$ and $\gamma=2$ and then use a suitable interpolation for $1<\gamma<2$. To this end, we start with the easy case $\gamma=1$. Let

$$
E_{\Omega_{m}} f\left(x, x_{n+1}, s\right)=\int_{\mathbb{S}^{n-1}} f\left(x-\phi(s) u, x_{n+1}-\psi(s)\right) \Omega_{m}(u) d \sigma(u) .
$$

If $f \in L^{\infty}\left(\mathbb{R}^{n+1}\right)$ and $h \in L^{1}\left(\mathbb{R}_{+}, d r / r\right)$, by Minkowski's inequality, we have

$$
\begin{aligned}
\left(\int_{0}^{\infty} \mid \int_{t / 2<|u| \leq t} f\right. & \left.\left.\left(x-\phi(|u|) u^{\prime}, x_{n+1}-\psi(|u|)\right) \frac{\Omega_{m}\left(u^{\prime}\right)}{|u|^{n-\varrho}} h(|u|) d u\right|^{2} \frac{d t}{t^{1+2 \sigma}}\right)^{1 / 2} \\
\leq & \left(\int_{0}^{\infty}\left(\int_{0}^{\infty}\left|E_{\Omega_{m}} f\left(x, x_{n+1}, s\right)\right||h(s)| \chi_{[t / 2, t]}(s) \frac{d s}{s^{1-\sigma}}\right)^{2} \frac{d t}{t^{1+2 \sigma}}\right)^{1 / 2}
\end{aligned}
$$




$$
\begin{aligned}
& \leq\left\|\Omega_{m}\right\|_{L^{1}\left(\mathbb{S}^{n-1}\right)}\|f\|_{L^{\infty}}\left(\int_{0}^{\infty}\left(\int_{0}^{\infty}|h(s)| \chi_{[t / 2, t]}(s) \frac{d s}{s^{1-\sigma}}\right)^{2} \frac{d t}{t^{1+2 \sigma}}\right)^{1 / 2} \\
& \leq\|f\|_{L^{\infty}} \int_{0}^{\infty}|h(s)|\left(\int_{s}^{2 s} \frac{d t}{t^{1+2 \sigma}}\right)^{1 / 2} \frac{d s}{s^{1-\sigma}} \leq C\|f\|_{L^{\infty}}\|h\|_{L^{1}\left(\mathbb{R}_{+}, d r / r\right)}
\end{aligned}
$$

for every $\left(x, x_{n+1}\right) \in \mathbb{R}^{n+1}$. Thus, by taking the supremum on both sides over all radial functions $h$ with $\|h\|_{L^{1}\left(\mathbb{R}_{+}, d r / r\right)} \leq 1$ we get

$$
\widetilde{M}_{\phi, \psi, \Omega_{m}}^{(1)} f\left(x, x_{n+1}\right) \leq C\|f\|_{L^{\infty}\left(\mathbb{R}^{n+1}\right)}
$$

for almost every $\left(x, x_{n+1}\right) \in \mathbb{R}^{n+1}$. Hence,

$$
\left\|\widetilde{M}_{\phi, \psi, \Omega_{m}}^{(1)}(f)\right\|_{L^{\infty}\left(\mathbb{R}^{n+1}\right)} \leq C\|f\|_{L^{\infty}\left(\mathbb{R}^{n+1}\right)} .
$$

The last inequality also yields Theorem 1.3(c).

Now we consider the case $\gamma=2$. By duality and a change of variable we have

$$
\begin{aligned}
& \widetilde{M}_{\phi, \psi, \Omega_{m}}^{(2)} f\left(x, x_{n+1}\right) \\
& \leq \sup _{\|h\|_{L^{2}\left(\mathbb{R}_{+}, d t / t\right)} \leq 1}\left(\int_{0}^{\infty}\left(\int_{0}^{\infty}|h(s)| \chi_{[t / 2, t]}(s)\left|E_{\Omega_{m}} f\left(x, x_{n+1}, s\right)\right|\right)^{2} \frac{d t}{t}\right)^{1 / 2} \\
& =\left(\int_{0}^{\infty} \int_{1 / 2}^{1}\left|E_{\Omega_{m}} f\left(x, x_{n+1}, s t\right)\right|^{2} \frac{d s d t}{s t}\right)^{1 / 2} \\
& =\left(\sum_{k \in \mathbb{Z}} \int_{a_{m}^{k}}^{a_{m}^{k+1}} \int_{1 / 2}^{1}\left|E_{\Omega_{m}} f\left(x, x_{n+1}, s t\right)\right|^{2} \frac{d s d t}{s t}\right)^{1 / 2} .
\end{aligned}
$$

For $k \in \mathbb{Z}$ and $m \in \mathbb{N}$, let $\theta_{m, k}=\phi\left(a_{m}^{k}\right)$. We notice that $\left\{\theta_{m, k}: k \in \mathbb{Z}\right\}$ is a lacunary sequence with $\theta_{m, k+1} / \theta_{m, k} \geq a_{m}>1$. Let $\left\{\psi_{k, m}\right\}_{k=-\infty}^{\infty}$ be a sequence in $C^{\infty}((0, \infty))$ such that

$$
0 \leq \psi_{k, m} \leq 1, \quad \sum_{k} \psi_{k, m}(t)=1
$$

$$
\operatorname{supp} \psi_{k, m} \subseteq\left[\theta_{m, k+1}^{-1}, \theta_{m, k-1}^{-1}\right], \quad\left|(d / d t)^{j} \psi_{k, m}(t)\right| \leq A_{j} / t^{j},
$$

where the constants $A_{j}$ are independent of $\theta_{m, k}$. Define an operator $T_{k, m}$ in $\mathbb{R}^{n+1}$ by $\left(\widehat{T_{k, m} f}\right)(\xi, \eta)=\psi_{k, m}(|\xi|) \widehat{f}(\xi, \eta)(\xi, \eta) \in \mathbb{R}^{n} \times \mathbb{R}$. Then for any $f \in \mathcal{S}\left(\mathbb{R}^{n+1}\right)$ and $l \in \mathbb{Z}$ we have $f\left(x, x_{n+1}\right)=\sum_{k \in \mathbb{Z}}\left(T_{k+l, m} f\right)\left(x, x_{n+1}\right)$. 
Thus, by (4.7) and Minkowski's inequality,

$$
\begin{aligned}
\widetilde{M}_{\phi, \psi, \Omega_{m}}^{(2)} f\left(x, x_{n+1}\right) & \leq\left(\sum_{k \in \mathbb{Z}} \int_{a_{m}^{k}}^{a_{m}^{k+1}} \int_{1 / 2}^{1}\left|\sum_{l \in \mathbb{Z}} Y_{k+l, t, s, m} f\left(x, x_{n+1}\right)\right|^{2} \frac{d s d t}{s t}\right)^{1 / 2} \\
& \leq \sum_{l \in \mathbb{Z}}\left(\sum_{k \in \mathbb{Z}} \int_{a_{m}^{k}}^{a_{m}^{k+1}} \int_{1 / 2}^{1}\left|Y_{k+l, t, s, m} f\left(x, x_{n+1}\right)\right|^{2} \frac{d s d t}{s t}\right)^{1 / 2} \\
& =\sum_{l \in \mathbb{Z}} X_{l, m} f\left(x, x_{n+1}\right),
\end{aligned}
$$

where

$$
\begin{aligned}
Y_{l, t, s, m} f\left(x, x_{n+1}\right) & =\int_{\mathbb{S}^{n-1}}\left(T_{l, m} f\right)\left(x-\phi(s t) u, x_{n+1}-\psi(s t)\right) \Omega_{m}(u) d \sigma(u), \\
X_{l, m} f\left(x, x_{n+1}\right) & =\left(\sum_{k \in \mathbb{Z}} \int_{a_{m}^{k}}^{a_{m}^{k+1}} \int_{1 / 2}^{1}\left|Y_{k+l, t, s, m} f\left(x, x_{n+1}\right)\right|^{2} \frac{d s d t}{s t}\right)^{1 / 2} .
\end{aligned}
$$

By the last inequality, we notice that (4.5) is proved for $\gamma=2$ if we can show that

$$
\left\|X_{l, m}(f)\right\|_{L^{p}\left(\mathbb{R}^{n+1}\right)} \leq C_{p}(m+1)^{1 / 2} 2^{-\delta_{p}|l|}\|f\|_{L^{p}\left(\mathbb{R}^{n+1}\right)}
$$

for some positive constants $C_{p}, \delta_{p}$ and for all $2 \leq p<\infty$. We start by proving (4.8) in the case $p=2$. By employing Plancherel's theorem, Fubini's theorem and Lemma 3.3 we obtain

$$
\begin{aligned}
\left\|X_{l, m}(f)\right\|_{2}^{2}= & \int_{\mathbb{R}^{n+1}} \sum_{k \in \mathbb{Z}} \int_{a_{m}^{k}}^{a_{m}^{k+1}} \int_{1 / 2}^{1}\left|Y_{k+l, t, s, m} f\left(x, x_{n+1}\right)\right|^{2} \frac{d s d t}{s t} d x d x_{n+1} \\
\leq & \sum_{k \in \mathbb{Z}} \int_{\mathbb{R}} \int_{\theta_{m, k+l+1}^{-1} \leq|\xi| \leq \theta_{m, k+l-1}^{-1}} \int_{a_{m}^{k}} \int_{1 / 2}^{a_{m}^{k+1}}|\widehat{f}(\xi, \eta)|^{2} \\
& \times\left|\int_{\mathbb{S}^{n-1}} \Omega_{m}(x) e^{-i(\phi(s t) \xi \cdot x+\eta \psi(t s))} d \sigma(x)\right|^{2} \frac{d s d t}{s t} d \xi d \eta \\
\leq & C(m+1) 2^{-2 \alpha|l|} \sum_{k \in \mathbb{Z} \mathbb{R}} \int_{\theta_{m, k+l+1}^{-1} \leq|\xi| \leq \theta_{m, k+l-1}^{-1}}|\widehat{f}(\xi, \eta)|^{2} d \xi d \eta \\
\leq & C(m+1) 2^{-2 \alpha|l|}\|f\|_{2}^{2},
\end{aligned}
$$

and hence

$$
\left\|X_{l, m}(f)\right\|_{2} \leq C(m+1)^{1 / 2} 2^{-\alpha|l|}\|f\|_{2} .
$$

Now we consider the case $p>2$. Choose $g$ in $L^{(p / 2)^{\prime}}\left(\mathbb{R}^{n+1}\right)$ with $\|g\|_{(p / 2)^{\prime}} \leq 1$ 
such that

$$
\begin{aligned}
\left\|X_{l, m}(f)\right\|_{p}^{2} & \\
= & \sum_{k \in \mathbb{Z}} \int_{\mathbb{R}^{n+1}} \int_{a_{m}^{k}}^{a_{m}^{k+1}} \int_{1 / 2}^{1}\left|Y_{k+l, t, s, m} f\left(x, x_{n+1}\right)\right|^{2} \frac{d s d t}{s t}\left|g\left(x, x_{n+1}\right)\right| d x d x_{n+1} \\
\leq & \sum_{k \in \mathbb{Z}} \int_{\mathbb{R}^{n+1}} \int_{a_{m}^{k}}^{a_{m}^{k+1}} \int_{1 / 2}^{1} \int_{\mathbb{S}^{n-1}}\left|\Omega_{m}(u)\right|\left|g\left(x+\phi(s t) u, x_{n+1}+\psi(s t)\right)\right| \\
& \times\left|T_{k+l, m} f\left(x, x_{n+1}\right)\right|^{2} d \sigma(u) \frac{d s d t}{s t} d x d x_{n+1} \\
\leq & C \sum_{k \in \mathbb{Z}} \int_{\mathbb{R}^{n+1}}\left|T_{k+l, m} f\left(x, x_{n+1}\right)\right|^{2} F_{m}^{*}(\widetilde{g})\left(-x,-x_{n+1}\right) d x d x_{n+1} \\
\leq & \left\|\sum_{k \in \mathbb{Z}}\left|T_{k+l, m} f\right|^{2}\right\|_{(p / 2)}\left\|F_{m}^{*}(\widetilde{g})\right\|_{(p / 2)^{\prime}},
\end{aligned}
$$

where $\widetilde{g}\left(x, x_{n+1}\right)=g\left(-x,-x_{n+1}\right)$. By using Lemma 3.6, the LittlewoodPaley theory and [St1, Theorem 3 along with the remark that follows its statement, p. 96], we have

$$
\left\|X_{l, m}(f)\right\|_{p} \leq C_{p}(m+1)^{1 / 2}\|f\|_{p} \quad \text { for } 2 \leq p<\infty .
$$

By interpolation between (4.9) and (4.10) we get (4.8), which ends the proof of (4.5) in the case $\gamma=2$.

Now, we handle the case $1<\gamma<2$. We shall use an idea employed in [Le]. By duality and a change of variable we have

$$
\widetilde{M}_{\phi, \psi, \Omega_{m}}^{(\gamma)} f\left(x, x_{n+1}\right) \leq\left(\int_{0}^{\infty}\left(\int_{1 / 2}^{1}\left|E_{\Omega_{m}} f\left(x, x_{n+1}, s t\right)\right|^{\gamma^{\prime}} \frac{d s}{s}\right)^{2 / \gamma^{\prime}} \frac{d t}{t}\right)^{1 / 2} .
$$

Thus,

$$
\left\|\widetilde{M}_{\phi, \psi, \Omega_{m}}^{(\gamma)} f\right\|_{L^{p}\left(\mathbb{R}^{n+1}\right)}=\|L(f)\|_{L^{p}\left(L^{2}\left(L^{\gamma^{\prime}}([1 / 2,1], d s / s), \mathbb{R}_{+}, d t / t\right), \mathbb{R}^{n+1}\right)},
$$

where $L: L^{p}\left(\mathbb{R}^{n+1}\right) \rightarrow L^{p}\left(L^{2}\left(L^{\gamma^{\prime}}([1 / 2,1], d s / s), \mathbb{R}_{+}, d t / t\right), \mathbb{R}^{n+1}\right)$ is defined by

$$
L(f)\left(x, x_{n+1}, t, s\right)=\int_{\mathbb{S}^{n-1}} f\left(x-\phi(s t) u, x_{n+1}-\psi(s t)\right) \Omega_{m}(u) d \sigma(u) .
$$

By (4.5) (for $\gamma=2$ ) and (4.6) (for $\gamma=1$ ), we find that

$$
\|L(f)\|_{L^{p}\left(L^{2}\left(L^{2}([1 / 2,1], d s / s), \mathbb{R}_{+}, d t / t\right), \mathbb{R}^{n+1}\right)} \leq C(m+1)^{1 / 2}\|f\|_{L^{p}\left(\mathbb{R}^{n+1}\right)}
$$

for $2<p<\infty$ and

$$
\|L(f)\|_{L^{\infty}\left(L^{2}\left(L^{\infty}([1 / 2,1], d s / s), \mathbb{R}_{+}, d t / t\right), \mathbb{R}^{n+1}\right)} \leq C\|f\|_{L^{\infty}\left(\mathbb{R}^{n+1}\right)} .
$$


Applying the real interpolation theorem for Lebesgue mixed normed spaces (see $[\mathrm{BP}]$ ), we conclude that

$$
\|L(f)\|_{L^{p}\left(L^{2}\left(L^{\gamma^{\prime}}([1 / 2,1], d s / s), \mathbb{R}_{+}, d t / t\right), \mathbb{R}^{n+1}\right)} \leq C(m+1)^{1 / \gamma^{\prime}}\|f\|_{L^{p}\left(\mathbb{R}^{n+1}\right)}
$$

for $\gamma^{\prime} \leq p<\infty$, which in turn implies (4.5) for $1<\gamma<2$. The proof of Theorem 3.1(a) and (c) is complete.

Proof of Theorem 1.3(b). Assume that $2<\gamma \leq \infty$ and $2<p<\infty$. As above, we have

$$
\widetilde{M}_{\phi, \psi, \Omega_{m}}^{(\gamma)} f\left(x, x_{n+1}\right) \leq\left(\int_{0}^{\infty}\left(\int_{1 / 2}^{1}\left|E_{\Omega_{m}} f\left(x, x_{n+1}, s t\right)\right|^{\gamma^{\prime}} \frac{d s}{s}\right)^{2 / \gamma^{\prime}} \frac{d t}{t}\right)^{1 / 2} .
$$

By applying the generalized Minkowski inequality, since $\gamma^{\prime}<2$ we get

$$
\widetilde{M}_{\phi, \psi, \Omega_{m}}^{(\gamma)} f\left(x, x_{n+1}\right) \leq\left(\int_{1 / 2}^{1}\left|H_{s, m} f\left(x, x_{n+1}\right)\right|^{\gamma^{\prime}} \frac{d s}{s}\right)^{1 / \gamma^{\prime}}
$$

where

$$
H_{s, m} f\left(x, x_{n+1}\right)=\left(\int_{0}^{\infty}\left|\int_{\mathbb{S}^{n-1}} f\left(x-\phi(s t) u, x_{n+1}-\psi(s t)\right) \Omega_{m}(u) d \sigma(u)\right|^{2} \frac{d t}{t}\right)^{1 / 2} .
$$

Now, we notice that Theorem 1.3(b) is proved once we prove that

$$
\left\|H_{s, m}(f)\right\|_{L^{p}\left(\mathbb{R}^{n+1}\right)} \leq C_{p}(m+1)^{1 / 2}\|f\|_{L^{p}\left(\mathbb{R}^{n+1}\right)}
$$

for $2<p<\infty$ and for some constant $C_{p}$ independent of $s$. To this end, by (4.11)-(4.12), applying the generalized Minkowski inequality and noticing that $1 \leq \gamma^{\prime}<2$ we get

$$
\begin{aligned}
\left\|\widetilde{M}_{\phi, \psi, \Omega_{m}}^{(\gamma)} f\right\|_{L^{p}\left(\mathbb{R}^{n+1}\right)} & \leq\left\|\int_{1 / 2}^{1}\left|H_{s, m}(f)\right|^{\gamma^{\prime}} \frac{d s}{s}\right\|_{L^{p / \gamma^{\prime}}\left(\mathbb{R}^{n+1}\right)}^{1 / \gamma^{\prime}} \\
& \leq\left(\int_{1 / 2}^{1}\left\|H_{s, m}(f)\right\|_{L^{p}\left(\mathbb{R}^{n+1}\right)}^{\gamma^{\prime}} \frac{d s}{s}\right)^{1 / \gamma^{\prime}} \\
& \leq C_{p}(m+1)^{1 / 2}\|f\|_{L^{p}\left(\mathbb{R}^{n+1}\right)}
\end{aligned}
$$

Let us now turn to the proof of (4.12). Let

$$
F_{m, k, s}(\xi, \eta)=\left(\int_{a_{m}^{k}}^{a_{m}^{k+1}}\left|\int_{\mathbb{S}^{n-1}} \Omega_{m}(x) e^{-i(\phi(s t) \xi \cdot x+\eta \psi(s t))} d \sigma(x)\right|^{2} \frac{d t}{t}\right)^{1 / 2} .
$$


By the proof of Lemma 3.2, it is easy to see that

$$
\begin{aligned}
\left|F_{m, k, s}(\xi, \eta)\right| & \leq C(m+1)^{1 / 2} \\
\left|F_{m, k, s}(\xi, \eta)\right| & \leq C(m+1)^{1 / 2}\left|\phi\left(a_{m}^{k-1}\right) \xi\right|^{-\alpha /(m+1)}, \\
\left|F_{m, k, s}(\xi, \eta)\right| & \leq C(m+1)^{1 / 2}\left|\phi\left(a_{m}^{k+1}\right) \xi\right|^{\alpha /(m+1)},
\end{aligned}
$$

for some constants $C$ and $\alpha$, where $(\xi, \eta) \in \mathbb{R}^{n} \times \mathbb{R}$. By (4.13)-(4.15), Lemma 3.7, using the partition of unity $\left\{\psi_{k, m}\right\}_{k=-\infty}^{\infty}$ and adapting a similar argument employed in the proof of Theorem 1.3(a) we get (4.12) (see also [A2]). The details will be omitted.

Proof of Theorem 1.2. Notice that

$$
\begin{aligned}
\mathcal{M}_{\Omega, \phi, \psi, h}^{\varrho} f\left(x, x_{n+1}\right) & \leq M_{\phi, \psi, \Omega}^{(\gamma)} f\left(x, x_{n+1}\right) \\
& =\sup _{\|h\|_{L \gamma\left(\mathbb{R}_{+}, d t / t\right)} \leq 1}\left|\mathcal{M}_{\Omega, \phi, \psi, h}^{\varrho} f\left(x, x_{n+1}\right)\right|
\end{aligned}
$$

and apply Theorem 1.3.

Proof of Theorem 1.1(a). Let us adopt the same notations as in the proof of Theorem 1.3. From (4.1) and (4.4) we can see that $\mathcal{M}_{\Omega, \phi, \psi, h}^{\varrho}(f) \leq$ $\sum_{m \in I \cup\{0\}} C_{m} \widetilde{\mathcal{M}}_{\Omega_{m}, \phi, \psi, h}^{\varrho}(f)$. Therefore, Theorem 1.1(a) is proved if we can show that

$$
\left\|\widetilde{\mathcal{M}}_{\Omega_{m}, \phi, \psi, h}^{\varrho}(f)\right\|_{L^{p}\left(\mathbb{R}^{n+1}\right)} \leq C_{p}(m+1)^{1 / 2}\|f\|_{L^{p}\left(\mathbb{R}^{n+1}\right)}
$$

for $m \in I \cup\{0\}$ and $2 \leq p<1 /(1 / 2-\alpha(\gamma))$, where $\alpha(\gamma)=\min \left\{1 / 2,1 / \gamma^{\prime}\right\}$. So let us prove (4.16). Since $\Delta_{\gamma}\left(\mathbb{R}_{+}\right) \subseteq \Delta_{2}\left(\mathbb{R}_{+}\right)$for $\gamma \geq 2$, we may assume that $1<\gamma \leq 2$ and $2 \leq p<2 \gamma /(2-\gamma)$. By Minkowski's inequality, it is easy to verify that $\widetilde{\mathcal{M}}_{\Omega_{m}, \phi, \psi, h}^{\varrho} f\left(x, x_{n+1}\right) \leq \sum_{j \in \mathbb{Z}} Q_{m, j} f\left(x, x_{n+1}\right)$, where

$$
Q_{m, j} f\left(x, x_{n+1}\right)=\left(\int_{0}^{\infty}\left|\sum_{k \in \mathbb{Z}} T_{k+j, m}\left(\sigma_{\widetilde{b}_{\mu}, t} * f\left(x, x_{n+1}\right)\right)\right|^{2} \frac{d t}{t}\right)^{1 / 2} .
$$

Therefore, to prove (4.16), it is enough to show that

$$
\left\|Q_{m, j}(f)\right\|_{L^{p}\left(\mathbb{R}^{n+1}\right)} \leq C_{p}(m+1)^{1 / 2} 2^{-\delta_{p}|j|}\|f\|_{L^{p}\left(\mathbb{R}^{n+1}\right)}
$$

for some $\delta_{p}>0$ and for $2 \leq p<2 \gamma /(2-\gamma)$. To this end, we first compute the $L^{2}$-norm of $Q_{m, j}(f)$. By Plancherel's theorem and the estimates (3.1)-(3.3) we have

and hence

$$
\left\|Q_{m, j}(f)\right\|_{L^{2}\left(\mathbb{R}^{n+1}\right)}^{2} \leq C(m+1) 2^{-\alpha|j|}\|f\|_{L^{2}\left(\mathbb{R}^{n+1}\right)}^{2}
$$

$$
\left\|Q_{m, j}(f)\right\|_{L^{2}\left(\mathbb{R}^{n+1}\right)} \leq C(m+1)^{1 / 2} 2^{-\alpha|j| / 2}\|f\|_{L^{2}\left(\mathbb{R}^{n+1}\right)} .
$$

Also, by Littlewood-Paley theory and Lemma 3.9 we have

$$
\left\|Q_{m, j}(f)\right\|_{L^{p}\left(\mathbb{R}^{n+1}\right)} \leq C_{p}(m+1)^{1 / 2}\|f\|_{L^{p}\left(\mathbb{R}^{n+1}\right)} \quad \text { for } 2 \leq p<\frac{2 \gamma}{2-\gamma} .
$$


By interpolating between (4.18) and (4.19) we get (4.17). This completes the proof of Theorem 1.1(a).

Now, as in the proof of Theorem 1.1(a), to prove Theorem 1.1(b)-(d), we need to obtain similar $L^{p}$ estimates for $Q_{m, j}(f)$ as in (4.19), and this can be achieved by applying respectively Lemmas 3.8, 3.10 and 3.11. Thus, Theorem 1.1 is proved.

\section{References}

[A1] H. M. Al-Qassem, Weighted $L^{p}$ boundedness for the function of Marcinkiewicz, Kyungpook Math. J. 46 (2006), 31-48.

[A2] - On the boundedness of maximal operators and singular operators with kernels in $L(\log L)^{\alpha}\left(S^{n-1}\right)$, J. Inequal. Appl. 2006, Art. ID 96732, 16 pp.

[AP1] H. Al-Qassem and Y. Pan, $L^{p}$ estimates for singular integrals with kernels belonging to certain block spaces, Rev. Mat. Iberoamer. 18 (2002), 701-730.

[AP2] - - , Singular integrals along surfaces of revolution with rough kernels, SUT J. Math. 39 (2003), 55-70.

[As] A. Al-Salman, On maximal functions with rough kernels in $L(\log L)^{1 / 2}\left(S^{n-1}\right)$, Collect. Math. 56 (2005), 47-56.

[AA] A. Al-Salman and H. M. Al-Qassem, Flat Marcinkiewicz integral operators, Turkish J. Math. 26 (2002), 329-338.

[AACP] A. Al-Salman, H. M. Al-Qassem, L. Cheng and Y. Pan, $L^{p}$ bounds for the function of Marcinkiewicz, Math. Res. Lett. 9 (2002), 697-700.

[AsP] A. Al-Salman and Y. Pan, Singular integrals with rough kernels in $L \log ^{+} L\left(\mathbf{S}^{n-1}\right)$, J. London Math. Soc. 66 (2002) 153-174.

[AAFJ] J. Ash, P. Ash, C. Fefferman and R. Jones, Singular integrals operators with complex homogeneity, Studia Math. 65 (1979), 31-50.

[BP] A. Benedek and R. Panzone, The spaces $L^{p}$, with mixed norm, Duke Math. J. 28 (1961), 301-324.

[CL] L. K. Chen and H. Lin, A maximal operator related to a class of singular integrals, Illinois J. Math. 34 (1990), 120-126.

[Di] Y. Ding, On Marcinkiewicz integral, in: Singular Integrals and Related Topics, III (Osaka, 2001), 28-38.

[DFP] Y. Ding, D. Fan and Y. Pan, On the $L^{p}$ boundedness of Marcinkiewicz integrals, Michigan Math. J. 50 (2002), 17-26.

[DLY] Y. Ding, S. Lu and K. Yabuta, A problem on rough parametric Marcinkiewicz functions, J. Austral. Math. Soc. 72 (2002), 13-21.

[DR] J. Duoandikoetxea and J. L. Rubio de Francia, Maximal functions and singular integral operators via Fourier transform estimates, Invent. Math. 84 (1986), 541-561.

[FP1] D. Fan and Y. Pan, Singular integral operators with rough kernels supported by subvarieties, Amer. J. Math. 119 (1997), 799-839.

[FP2] - - - A singular integral operators with rough kernel, Proc. Amer. Math. Soc. 125 (1997), 3695-3703.

[FPY] D. Fan, Y. Pan and D. Yang, A weighted norm inequality for rough singular integrals, Tohoku Math. J. 51 (1999), 141-161.

[Ho] L. Hörmander, Translation invariant operators, Acta Math. 104 (1960), 93-139. 
[GR] J. García-Cuerva and J. L. Rubio de Francia, Weighted Norm Inequalities and Related Topics, North-Holland, 1985.

[KWWZ] W. Kim, S. Wainger, J. Wright and S. Ziesler, Singular integrals and maximal functions associated to surfaces of revolution, Bull. London Math. Soc. 28 (1996), 291-296.

[Le] H. V. Le, Maximal operators and singular integral operators along submanifolds, J. Math. Anal. Appl. 296 (2004), 44-64.

[SY] M. Sakamoto and K. Yabuta, Boundedness of Marcinkiewicz functions, Studia Math. 135 (1999), 103-142.

[Sa] S. Sato, Remarks on square functions in the Littlewood-Paley theory, Bull. Austral. Math. Soc. 58 (1998), 199-211.

[St1] E. M. Stein, On the functions of Littlewood-Paley, Lusin and Marcinkiewicz, Trans. Amer. Math. Soc. 88 (1958), 430-466.

[St2] - Singular Integrals and Differentiability Properties of Functions, Princeton Univ. Press, Princeton, NJ, 1970.

[TW] A. Torchinsky and S. Wang, A note on the Marcinkiewicz integral, Colloq. Math. 61-62 (1990), 235-243.

[Wa] T. Walsh, On the function of Marcinkiewicz, Studia Math. 44 (1972), 203-217.

Department of Mathematics and Physics

Qatar University

Doha, Qatar

E-mail: husseink@yu.edu.jo, husseink@qu.edu.qa
Department of Mathematics

University of Pittsburgh Pittsburgh, PA 15260, U.S.A.

E-mail: yibiao@pitt.edu

Received August 22, 2007

Revised version September 3, 2008 